\title{
Renormalization Group and Critical Phenomena. II. Phase-Space Cell Analysis of Critical Behavior*
}

\author{
Kenneth G. Wilson \\ Laboratory of Nuclear Studies, Cornell University, Ithaca, New York 14850 \\ (Received 2 June 1971)
}

\begin{abstract}
A generalization of the Ising model is solved, qualitatively, for its critical behavior. In the generalization the spin $s_{\overrightarrow{\mathrm{n}}}$ at a lattice site $\overrightarrow{\mathrm{n}}$ can take on any value from $-\infty$ to $\infty$. The interaction contains a quartic term in order not to be pure Gaussian. The interaction is investigated by making a change of variable $s_{\mathrm{i}}=\sum_{m} \psi_{m}(\mathrm{n}) s_{m}^{\prime}$, where the functions $\psi_{m}(\overrightarrow{\mathrm{n}})$ are localized wavepacket functions, There are a set of orthogonal wave-packet functions for each order-of-magnitude range of the momentum $\vec{k}$. An effective interaction is defined by integrating out the wave-packet variables with momentum of order 1 , leaving unintegrated the variables with momentum <0.5. Then the variables with momentum between 0.25 and 0.5 are integrated, etc. The integrals are computed qualitatively. The result is to give a recursion formula for a sequence of effective Landau-Ginsberg-type interactions. Solution of the recursion formula gives the following exponents: $\eta=0, \gamma=1.22, \nu=0.61$ for three dimensions. In five dimensions or higher one gets $\eta=0, \gamma=1$, and $\nu=\frac{1}{2}$, as in the Gaussian model (at least for a small quartic term). Small corrections neglected in the analysis may make changes (probably small) in the exponents for three dimensions.
\end{abstract}

\section{INTRODUCTION}

In Paper I of this series ${ }^{1}$ the Kadanoff picture of scaling $^{2}$ for the Ising model was discussed. Kadanoff considered the problem of the critical behavior of the Ising model. ${ }^{3}$ He proposed that the critical behavior could be understood in terms of the effective interactions between blocks of spins of block size $L$, with $L$ being larger than 1 but smaller than the correlation length $\xi$. Kadanoff suggested that one could introduce an effective spin variable $s_{m}^{\prime}$ for each block and that the effective interactions of these spins would have the Ising form, but with interaction parameters ${ }^{4} K_{L}$ and $h_{L}$ depending on $L$. In $I$ it was suggested that a basic assumption of the Kadanoff theory is an analyticity assumption, namely, that $K_{n L}$ and $h_{n L}$ depend analytically on $K_{L}$ and $h_{L}$ if $n$ is a fixed integer. The rationale for this is that a block of spins of size $n L$ is made up of a finite number of blocks of size $L$, so the sums over the interactions of the $L$-size blocks which give the interaction on one $n L$-size block ought to be analytic. (In I this assumption was stretched to include the case that $n=1+\epsilon$, where $\epsilon$ is infinitesimal. )

No justification has been found for the Kadanoff picture. The difficulty is that Kadanoff assumes that the effective spin variable $s_{m}^{\prime}$ has only two values, up or down. In the exact Ising model a block of spins of size $L$ and $z^{L^{d}}$ different configurations (where $d$ is the dimensionality of the system) and it is hard to see how to reduce the $z^{L^{d}}$ different configurations to just two. Because there has been no justification for the Kadanoff picture, it has been impossible to calculate specific critical ex- ponents within the Kadanoff picture; the best one can do is to derive the scaling laws ${ }^{3}$ which relate all the critical exponents to two unknown parameters.

Is there some generalization of the Kadanoff picture which can be derived from the exact partition function of the Ising model? This problem will be discussed, qualitatively, in this paper. It will be shown here that one can obtain an effective interaction for blocks of spins of size $L$, but the effective interaction will not be simply an Ising interaction. The effective interactions discussed in this paper differ from Kadanoff's effective Ising models in several respects. The effective block spins $s_{m}^{\prime}$ of Kadanoff's theory will be replaced by a field variable $s_{L}(\overrightarrow{\mathrm{x}})$. The box size $L$ will be built into the field $s_{L}(\overrightarrow{\mathrm{x}})$ in a qualitative sense, namely, $s_{L}(\overrightarrow{\mathrm{x}})$ will be restricted to have wavelengths $\geq L$ only. This means that $s_{L}(\overrightarrow{\mathrm{x}})$ does not vary enormously within a block of size $L$ and for qualitative purposes one can think of $s_{L}(\overrightarrow{\mathbf{x}})$ within a block as if it were a single block variable. Also, in contrast to Kadanoff's picture, the magnitude of $s_{L}(\overrightarrow{\mathrm{x}})$ will be unrestricted. The effective interaction of this paper has the Landau-Ginsberg form ${ }^{5}$ :

$$
\mathfrak{H}_{L}=-\frac{1}{2} K_{L} \int_{\overrightarrow{\mathbf{x}}} \nabla s_{L}(\overrightarrow{\mathrm{x}})^{2}-\int_{\overrightarrow{\mathbf{x}}} P_{L}\left[s_{L}(\overrightarrow{\mathrm{x}})\right],
$$

where $\int_{\overrightarrow{\mathrm{x}}}$ means $\int d^{d} x, K_{L}$ is a constant, and $P_{L}[s]$ is a function which changes with $L$. The function $P_{L}[s]$ is equivalent to an infinite number of parameters (for example, $P_{L}[s]$ could be represented by the coefficients of its Taylor series expansion in $s)$. So instead of having just two parameters $K_{L}$ and $h_{L}$ depending on the block size $L$, one now has an infinite number of $L$-dependent parameters. In 
the language of I, this means one has an infinite number of "irrelevant variables." 6

A recursion formula will be derived which determines $P_{2 L}[s]$ given the function $P_{L}[s]$. The critical behavior of the Ising model will be obtained, qualitatively, by solving the recursion formula. It will be found that at the critical point the functions $P_{L}[s]$ approach a limit as $L \rightarrow \infty$ except for some scale factors. This limit is analogous to the critical "saddle point" discussed in I for the case of one irrelevant variable. Solutions of the recursion formula near the critical point will show the same qualitative features as discussed in I for the case of one irrelevant variable. From these solutions three critical exponents will be calculated explicitly (for three dimensions). The results are $\nu=0.61$, $\gamma=1.22$, and $\eta=0 .{ }^{7}$ These are qualitative results: Corrections to the analysis of this paper can change these exponents, but hopefully not by much.

It will also be found that in five dimensions or higher these exponents have the mean field values $\nu=0.5, \gamma=1$, and $\eta=0$. Four dimensions will be seen to be the dividing point below which the exponents are nonclassical; no exponents will be obtained for four dimensions.

The ideas which underlie the recursion formula are as follows. The partition function will be expressed as a functional integral over all functions $s_{L}(\overrightarrow{\mathrm{x}})$ of the interaction $e^{3 C_{L}}$. This is in lieu of the sum over all block spin variables which appears in the Kadanoff picture. The limitation on wavelengths in $s_{L}(\overrightarrow{\mathrm{x}})$ means the Fourier transform of $s_{L}(\overrightarrow{\mathrm{x}})$, say $\sigma_{L}(\overrightarrow{\mathrm{k}})$, must be zero for $|\overrightarrow{\mathrm{k}}|>L^{-1}$. To be precise the functional integral is over all functions $\sigma_{L}(\overrightarrow{\mathrm{k}})$ defined for $|\overrightarrow{\mathrm{k}}|<L^{-1}$. To derive the recursion formula, the variables $\sigma_{L}(\overrightarrow{\mathrm{k}})$ for $0.5 L^{-1}<|\overrightarrow{\mathrm{k}}|<L^{-1}$ will be integrated out. When these integrations are completed, one is left with a functional integral over all functions $\sigma_{L}(\overrightarrow{\mathrm{k}})$ defined for $0<|\overrightarrow{\mathrm{k}}|<0.5 L^{-1}$. The Fourier transform of such functions can be denoted $s_{2 L}(\overrightarrow{\mathrm{x}})$ since they contain only wavelengths $\geq 2 L$. The integrand of this new functional integral will be written in the form $e^{3 C_{2 L}}$; this defines the interaction $\mathfrak{H}_{2 L}$.

The evaluation of the integrals will be done qualitatively, not quantitatively; an accurate calculation would give more complicated effective interactions $\mathfrak{H}_{L}$ than the Landau-Ginsberg form (complications include more derivative terms, even nonlocal interactions).

The analysis given in this paper is based on an earlier discussion of a problem in quantum field theory (pions interacting with a fixed source). ${ }^{8}$ The ideas of this paper can be applied to relativistic quantum field theory. ${ }^{9}$ So the ideas of this paper are not special to the problem of critical phenomena. The basic problem causing the difficulties in understanding critical phenomena is the problem of the infinite number of degrees of freedom. This problem is also the bottleneck in quantum field theory and in many of the stubborn problems of solid-state physics. The methods of this paper are methods for attacking the problem of the infinite number of degrees of freedom regardless of where this problem arises.

The model that will be studied in this paper is a generalization of the Ising model. Consider the partition function

$$
\begin{gathered}
Z(K, h ; r, \lambda)=\Pi_{\overrightarrow{\mathrm{n}}} \int_{-\infty}^{\infty} d s_{\overrightarrow{\mathrm{n}}} \exp \left(-r \sum_{\overrightarrow{\mathrm{n}}} s_{\overrightarrow{\mathrm{n}}}^{2}-\lambda \sum_{\overrightarrow{\mathrm{n}}} s_{\overrightarrow{\mathrm{n}}}^{4}\right. \\
\left.+K \sum_{\overrightarrow{\mathrm{n}}} \sum_{i} s_{\overrightarrow{\mathrm{n}}} S_{\overrightarrow{\mathrm{n}}+\hat{i}}+h \sum_{\overrightarrow{\mathrm{n}}} s_{\overrightarrow{\mathrm{n}}}\right) .
\end{gathered}
$$

The vectors $\vec{n}$ are vectors with integral components. They label the lattice sites of a simple plane, cubic, or hypercubic lattice. The $\sum_{i}$ is a sum over the $d$ axes of the lattice $(d=2$ for a plane lattice, $d=3$ for a cubic lattice, etc.). A unit vector along the axis $i$ is denoted $\hat{i}$. The changes from the Ising model are that $s_{\text {if }}$ is a continuous spin variable, instead of being \pm 1 only, and that terms proportional to $s_{\overline{\mathrm{I}}}^{2}$ and $s_{\mathrm{I}}^{4}$ have been added in the exponential. Let $K$ and $h$ be the ordinary thermodynamic variables $(K=-J / k T$ and $h=-\mu H / k T$, where $J$ is a spin-spin coupling parameter, $\mu$ the magnetic moment of a spin, and $H$ the external magnetic field). The constants $r$ and $\lambda$ are to be fixed constants independent of $T$ and $H$. The constants $r$ and $\lambda$ determine the intrinsic probability for the spin $s_{\vec{n}}$ to have any particular value. This intrinsic probability is essential in the continuousspin case since otherwise the integrals over $s_{\vec{n}}$ will diverge. If $\lambda=0$ the partition function reduces to the Gaussian model of Berlin and Kac, ${ }^{10}$ which has special critical exponents disagreeing both with experiment and with numerical calculations for the Ising model. For $\lambda \rightarrow \infty$ and $r \rightarrow-\infty$ the model reduces to the Ising model, as follows. If the intrinsic probability function for the spin $s_{\vec{a}}$ has the form $\exp \left[-\lambda\left(s_{n}^{2}-1\right)^{2}+\lambda\right]$ (i. e., $\left.r=-2 \lambda\right)$, then it has $\max -$ ima at $s_{\overrightarrow{\mathrm{n}}}=+1$ and -1 , and if $\lambda$ is large the probability function is very sharply peaked at $s_{\overrightarrow{\mathrm{n}}}= \pm 1$ (see Fig. 1). The partition function of Eq. (1.2) for general $\lambda$ interpolates between the Gaussian model and the Ising model. A very similar model has been discussed by Migdal $^{9}$ and another similar model by Langer. ${ }^{10}$

One needs a term besides $-r s_{\mathrm{n}}^{2}$ in the probability function in order to get departures from the Gaussian model. The use of an $s_{\mathbf{n}}^{4}$ term in particular is arbitrary; one can use any function of $s_{\mathbb{\mathrm { n }}}^{2}$ instead which is nonlinear in $s_{\overrightarrow{\mathrm{n}}}^{2}$ and which does not destroy the normalizability of the probability function (e.g. , $-\lambda s_{\mathrm{in}}^{4}$ is all right but $+\lambda s_{\overrightarrow{\mathrm{n}}}^{4}$ is not, for positive $\lambda$ ).

There are two reasons why a continuous spin is used in this paper instead of a discrete spin. One 


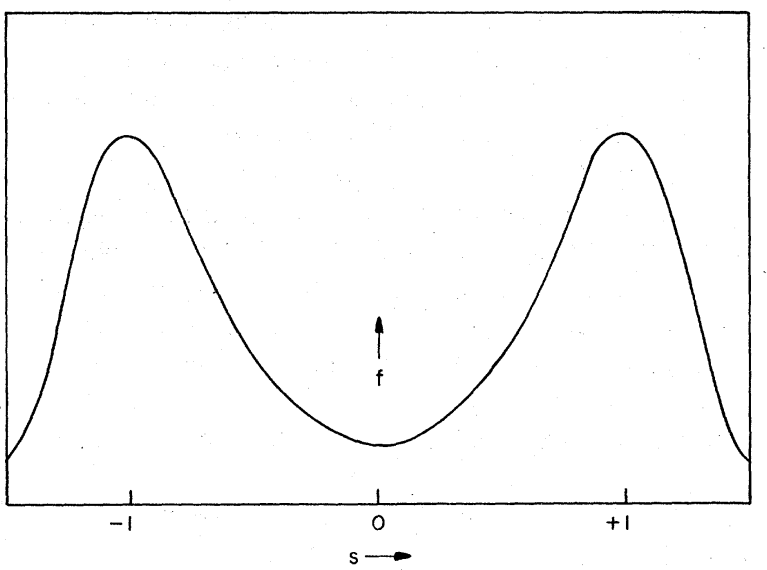

FIG. 1. Plot of the function $f(s)=e^{-2 \lambda s^{2}+2 \lambda s}$ vs $s$ for $\lambda=1.8$.

is a technical one: The analysis of this paper makes use of changes of variables to linear combinations of the $s_{\vec{n}}$ and these changes are awkward to make if $s_{\vec{i}}$ is restricted to be \pm 1 . The second reason is that the analysis of this paper predicts that the critical exponents in five dimensions and higher will be the same as the exponents of the Gaussian model, at least for small $\lambda$, and to show this the model must include the Gaussian model as a special case.

The lattice variable $s_{\overrightarrow{\mathrm{I}}}$ will be replaced by a field $s(\overrightarrow{\mathrm{x}})$ with the Fourier components of $s(\overrightarrow{\mathrm{x}})$ restricted to have momenta of order 1 or less. This replacement is made because the problems of critical phenomena concern large wavelengths for which the lattice variable $\vec{n}$ should be equivalent to a continuous variable $\vec{x}$. Once this replacement is made the interaction of Eq. (1.2) can be expressed in the Landau-Ginsberg form.

To carry out the functional integral over the variables $\sigma_{L}(\overrightarrow{\mathrm{k}})$ for $0.5 L^{-1}<|\overrightarrow{\mathrm{k}}|<L^{-1}$, a change of variable will be made replacing the functional variable $\sigma_{L}(\overrightarrow{\mathrm{k}})$ by an infinite set of discrete variables $s_{L \overrightarrow{\mathrm{m}}}$. The change of variables is set up by expanding the function $\sigma_{L}(\overrightarrow{\mathrm{k}})$ in terms of an orthonormal set of wave packets defined for $0.5 L^{-1}<|\vec{k}|<L^{-1}$. These wave packets will not be written down explicitly; rather their qualitative features will be determined from phase-space arguments based on an analogy with the phase-space formulation of Fermi statistics.

In Sec. II a preliminary discussion is presented of the wave-packet ("phase-space cell") variables $s_{L \overrightarrow{\mathrm{m}}}$ and their usefulness in studying critical phenomena. In particular, the form of the interaction of Eq. (1.2) in terms of phase-space cell variables is discussed and a preliminary estimate of the order of magnitudes of various terms in the inter- action is given. (In Sec. II, and thereafter, the effective lattice spacing variable $L$ will be replaced by a variable $l$, given by $L=2^{l}$.) In Sec. III the full interaction is analyzed in terms of phase-space cell variables and the recursion formula for the effective Landau-Ginsberg interactions is derived. In Sec. IV solutions of the recursion formulas are discussed; both analytic approximations and numerical calculations are reported, and values for the exponents $\gamma, \eta$, and $\nu$ are obtained. In Sec. V various limitations of the present work are discussed, including the reasons for omitting the case of a finite magnetic field, temperatures below the critical temperature, or two dimensions. Section VI concludes this paper with a discussion of the ideas learned from the phase-space cell analysis.

\section{ANALYSIS OF PARTITION FUNCTION: PRELIMINARY DISCUSSION}

\section{A. Approximate Factorization and Wave-Packet Variables}

It would be ideal if one could introduce a set of integration variables in the integral of Eq. (1.2) such that the integrand factorizes. For example, if $K$ is zero the integrand factorizes into separate functions for each $s_{\vec{n}}$ and the partition function is an infinite product of independent single-variable integrals. If $\lambda=0$ the integrand factorizes in terms of the Fourier-transform variables

$$
\sigma_{\overrightarrow{\mathbf{R}}}=\sum_{\overrightarrow{\mathrm{n}}} e^{i \overrightarrow{\mathbf{k}} \cdot \overrightarrow{\mathbf{n}}} s_{\overrightarrow{\mathbf{n}}} .
$$

In this case one has

$$
\begin{aligned}
-r & \sum_{\overrightarrow{\mathbf{i}}} s_{\overrightarrow{\mathbf{n}}}^{2}+K \sum_{\overrightarrow{\mathbf{i}}} \sum_{i} s_{\overrightarrow{\mathbf{n}}} s_{\overrightarrow{\mathbf{n}}+\hat{i}}+h \sum_{\overrightarrow{\mathbf{n}}} s_{\overrightarrow{\mathbf{n}}} \\
& =-r \int_{\overrightarrow{\mathbf{k}}} \sigma_{\overrightarrow{\mathbf{k}}} \sigma_{-\overrightarrow{\mathbf{k}}}+K \int_{\overrightarrow{\mathbf{k}}} \sum_{i} \cos k_{i} \sigma_{\overrightarrow{\mathbf{k}}} \sigma_{-\overrightarrow{\mathbf{k}}}+h \sigma_{0}
\end{aligned}
$$

(where $\int_{\overrightarrow{\mathrm{x}}}$ means $\Pi_{i}(2 \pi)^{-1} \int_{-\pi}^{\pi} d k_{i}$ and $k_{i}$ is a component of $\vec{k})$, so each pair of variables $\left(\sigma_{\vec{k}}, \sigma_{-\vec{k}}\right)$ is independent of the corresponding pairs with different momenta. With both $K$ and $\lambda$ nonzero there is a conflict between the $K$ and $\lambda$ terms in the interaction which prevents factorization; for any choice of integration variables. However, one can try to achieve an approximate factorization: namely, one can try to introduce a change of variables such that the coupling between different degrees of freedom is smaller than the terms involving only a single degree of freedom.

Imagine expanding the function $s_{\overrightarrow{\mathrm{n}}}$ (as a function of the discrete variable $\vec{n}$ ) in a complete set of wave functions $\psi_{m}(\overrightarrow{\mathrm{n}})$ :

$$
s_{\overrightarrow{\mathrm{n}}}=\sum_{m} \psi_{m}(\overrightarrow{\mathrm{n}}) s_{m}^{\prime} .
$$

This expansion defines a change of variables, from the variables $s_{\text {in }}$ to the variables $s_{m}^{\prime}$. Let the wave 
functions $\psi_{m}(\overrightarrow{\mathbf{n}})$ be orthonormal (and real so that the variables $s_{m}^{\prime}$ are real); orthonormality means that

$$
\Sigma_{\mathrm{i}} \psi_{m}(\overrightarrow{\mathrm{n}}) \psi_{m_{1}}(\overrightarrow{\mathrm{n}})=\delta_{m m_{1}} \text {. }
$$

Let the Fourier transform of $\psi_{m}(\overrightarrow{\mathrm{n}})$ be $\phi_{m}(\overrightarrow{\mathrm{k}})$ :

$$
\phi_{m}(\overrightarrow{\mathbf{k}})=\Sigma_{\overrightarrow{\mathbf{n}}} e^{i \overrightarrow{\mathbf{k}} \cdot \overrightarrow{\mathbf{n}}} \psi_{m}(\overrightarrow{\mathrm{n}})
$$

so that the Fourier transform of $s_{\overrightarrow{\mathbb{R}}}$ is $\sigma_{\overrightarrow{\mathbf{r}}}$ with

$$
\sigma_{\overrightarrow{\mathbf{k}}}=\sum_{m} \phi_{m}(\overrightarrow{\mathbf{k}}) s_{m}^{\prime} \text {. }
$$

One would like to keep the width of the functions $\phi_{m}(\overrightarrow{\mathbf{k}})$ small for doing so will minimize coupling of different degrees of freedom ( $m$ and $m_{1}$, say) due to the $K$ term. From Eq. (2.2), the part of the $K$ term coupling $m$ to $m_{1}$ has the form

$$
K \int_{\overrightarrow{\mathbf{k}}} \sum_{i} \cos \left(k_{i}\right) \phi_{m}(\overrightarrow{\mathrm{k}}) \phi_{m_{1}}(-\overrightarrow{\mathrm{k}}) s_{m}^{\prime} s_{m_{1}}^{\prime} .
$$

If the wave packets limit the range of $\vec{k}$ in the integral enough, one can approximate $\cos k_{i}$ by the constant $\cos k_{i M}$ where $k_{i M}$ is the mean momentum of one of the packets. However, $\int_{\vec{k}} \phi_{m}(\vec{k}) \phi_{m_{1}}(-\vec{k})$ is the orthogonality integral expressed in terms of the momentum-space wave functions, so this vanishes unless $m=m_{1}$. Thus the nonvanishing part of the coupling comes only from the integral

$$
K \int_{\overrightarrow{\mathbf{k}}}\left(\cos k_{i}-\cos k_{i M}\right) \phi_{m}(\overrightarrow{\mathbf{k}}) \phi_{m_{1}}(-\overrightarrow{\mathrm{k}}) s_{m}^{\prime} s_{m_{1}}^{\prime}
$$

and this is small if $k_{i}$ cannot differ much from $k_{i M}$ over the width of the packets $\phi_{m}(\overrightarrow{\mathrm{k}})$ and $\phi_{m_{1}}(-\overrightarrow{\mathrm{k}})$.

In conflict with the desire to keep the width of $\phi_{m}(\overrightarrow{\mathrm{k}})$ small is the desire to keep the width of $\psi_{m}(\overrightarrow{\mathrm{n}})$ small, for if these wave packets cover many lattice sites then there will be many wave packets which overlap at a given site $\vec{n}$ and then there will be large couplings of different degrees of freedom through the $\lambda \dot{s}_{\mathrm{n}}^{4}$ terms. Evidently one wants the wave packets $\psi_{m}(\overrightarrow{\mathrm{n}})$ and $\phi_{m}(\overrightarrow{\mathbf{k}})$ to be minimal wave packets, i.e., the widths $\Delta n$ and $\Delta k$ should satisfy the lower bound imposed by the uncertainty principle:

$$
\Delta n \Delta k \sim 1 .
$$

(The exact bound is unimportant as the analysis given here will be only an order of magnitude analysis. )

There is a useful qualitative characterization of a complete set of minimal orthonormal wavepackets which is suggested by the layman's description of Fermi statistics. If one wants to obtain the principal qualitative predictions of Fermi statistics without discussing antisymmetrized wave functions, one instead describes the electrons as classical particles but subject to the restriction that no more than one electron (with a given spin) can occupy a unit volume in phase space. One then divides phase space into cells of unit volume each of which can contain at most one electron of a given spin. When this picture is translated into the language of wave functions, it is evident that one associates a minimal wave packet with each cell of unit volume in phase space. Two different wave packets are orthogonal if the corresponding cells in phase space do not overlap; a set of wave packets are complete if the corresponding cells fill the whole of phase space. For qualitative purposes, one can treat the magnitude of $\left|\phi_{m}(\vec{k})\right|$ as constant if $\vec{k}$ is within the range of the corresponding cell ${ }^{11}$ and 0 elsewhere, and similarly for $\left|\psi_{m}(\overrightarrow{\mathrm{n}})\right|$ (see Fig. 2). Finally the magnitudes of $\left|\phi_{m}(\overrightarrow{\mathbf{k}})\right|$ and $\left|\psi_{m}(\overrightarrow{\mathrm{n}})\right|$ are determined by the normalization condition. If $V_{x}(m)$ and $V_{k}(m)$ are the momentum-space volume and position-space volume, respectively, for the phase-space cell $m\left(V_{x}=V_{k}^{-1}\right.$ so that the phase-space volume is 1 ), then one has

$\left|\phi_{m}(\overrightarrow{\mathbf{k}})\right| \sim\left[V_{k}(m)\right]^{-1 / 2}$ (within phase-space cell $m$ ),

$\left|\psi_{m}(\overrightarrow{\mathrm{n}})\right| \sim\left[V_{x}(m)\right]^{-1 / 2}$ (within phase-space cell $m$ ).

This is a qualitative characterization of a complete orthonormal set of minimal wave packets. For quantitative purposes one would have to take into account tails of the wave packets which extend outside their assigned cells.

It will be assumed here that one can divide phase space into cells of unit volume in any way one pleases and still be able to construct a corresponding set of minimal wave packets. There is no guarantee that this is actually possible, and no examples of such a set of wave packets will be given here. The analogy to the qualitative description of Fermi statistics should be sufficient to justify the qualitative analysis given here.

\section{B. Phase-Space Decomposition}

The problem of how best to define the degrees of freedom $s_{m}^{\prime}$ is now the problem of finding the best division of phase space into cells of unit volume. We propose the following solution. The variable $|\vec{k}|$ has a range from 0 to of order 1 ; divide this interval logarithmically, namely, define the intervals

$$
2^{-l} \leq|\vec{k}| \leq 2 \times 2^{-l}
$$

as the momentum-space division. This means momentum space is divided into spherical shells whose inner radius is half the outer radius. The largest shell is the shell $l=0(1 \leq|\overrightarrow{\mathrm{k}}| \leq 2)$ (in this qualitative analysis we ignore the range $|\vec{k}|>2$ up to the maximum value which is of order $\pi$ ). There are an infinite number of smaller shells. For each shell in momentum space separately one divides position space into blocks of equal size forming a cubic 


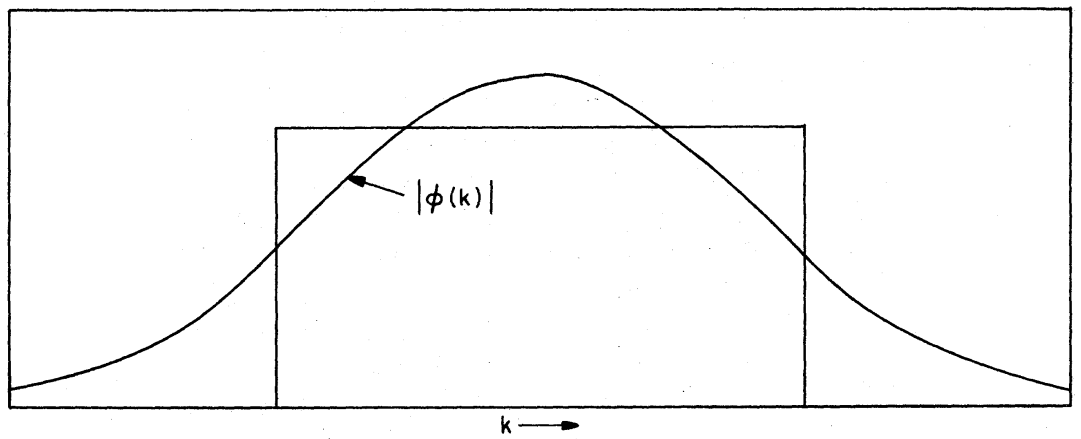

FIG. 2. Example of a wave-packet $|\phi(k)|$ (curve shown) and a squarewave approximation to $|\phi(k)|$ used for order-of-magnitude calculations.

lattice, each block having volume inverse to the volume of the momentum-space shell. This requirement on the position-space blocks means that the $l$ th momentum-space shell has corresponding position-space blocks whose lengths are of order $2^{l}$. The position-space blocks double in length each time $l$ is increased by 1 . This division of phase space is illustrated in Fig. 3.

The motivation for this method of division is the following. The momentum-space intervals have been kept small enough so that the coupling terms coming from the nearest-neighbor spin interaction are smaller by about a factor of 2 than the corresponding diagonal terms [diagonal terms involve

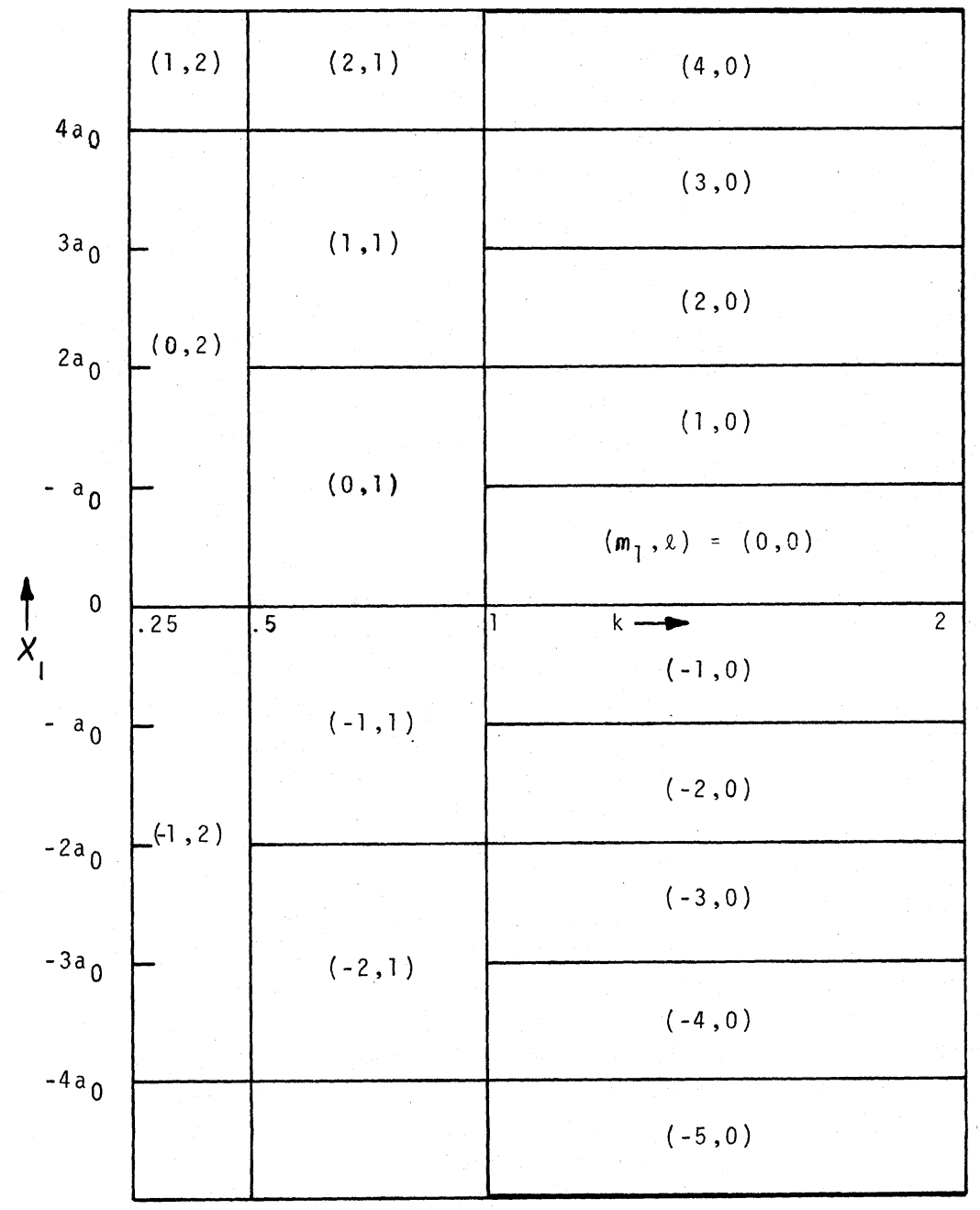

FIG. 3. Division of phase space into cells. The magnitude of $\vec{k}$ is plotted versus one component of $x$. The range $0.25<|\vec{k}|<2$ is shown. Each phase-space cell is labeled with the corresponding values of $m_{1}$ and $l$. 
$\left(s_{m}^{\prime}\right)^{2}$ not $\left.s_{m}^{\prime} s_{m_{1}}^{\prime}\right]$. It appears at first sight that the coupling terms for the large- $l$ shells are very much smaller than the diagonal terms, but this is an illusion. The reason is that the diagonal terms coming from the $K \sum_{\hat{\mathbf{t}}} s_{\hat{\mathrm{i}}} s_{\hat{\mathrm{i}}+\hat{i}}$ term tend to cancel the corresponding terms coming from $-r \sum_{\mathrm{if}} s_{\mathrm{in}}^{2}$. If one combines these two terms, then in momentum space one is looking at $\int_{\overrightarrow{\mathrm{k}}}\left[-r+K \sum_{i} \cos k_{i}\right] \sigma_{\overrightarrow{\mathbf{k}}} \sigma_{-\overrightarrow{\mathrm{k}}}$. At the critical point the coefficient $\left[-r+K \sum_{i} \cos k_{i}\right]$ will vanish (to a first approximation; see later) for $\overrightarrow{\mathrm{k}}=0$, and for nonzero $k_{i}$ the coefficient is of order $-K k^{2} / 2$, where $k^{2}=\sum_{i} k_{i}^{2}$. It is still true that the coupling terms in a given shell (i.e., coupling of different position-space blocks for a given momentum shell) are smaller than the diagonal terms, but only by about a factor 2 , since the variation in $k^{2}$ over a shell is roughly half the mean value of $k^{2}$ in a shell. If we had allowed a much larger range of $k^{2}$ in a shell the size of the coupling terms would have become essentially equal to the diagonal terms -an intolerable situation.

The coupling terms in the $\lambda \sum_{\text {in }} s_{\mathrm{in}}^{4}$ term are reduced in size in the proposed division by the following mechanism. The degrees of freedom which couple through this term are degrees of freedom from different momentum-space shells whose positionspace blocks overlap. A coupling term will thus involve at least two position-space blocks corresponding to two different momentum-space shells. These blocks will be disparate in size, one being several times larger than the other (at least twice as large). The coupling term will involve products of the large-block wave packet times the smallblock wave packet, summed over the lattice sites contained in the small block. The wave packet for the large block is smaller in size than the wave packet for the small block because of the normalization condition on these wave packets. Hence the coupling term is small at least compared to the diagonal term for the small block since the only difference is the substitution of a large-block wave packet for a small-block wave packet.

In case the above argument is not clear, here is an example worked out in detail. Label the new variables $s_{m}^{\prime}$ as $^{12} s_{\overrightarrow{\mathrm{m}} l}$, where $l$ labels the momentum-space shell and $\vec{m}$ is a lattice variable distinguishing the blocks in position space associated with a given shell (see Fig. 3). Compare the coupling term proportional to $s_{0 l}^{2} s_{00}^{2}$ to the diagonal terms $s_{0 l}^{4}$ and $s_{00}^{4}$. These terms are, respectively,

$$
\begin{aligned}
& -6 \lambda \sum_{\overrightarrow{\mathrm{n}}} \psi_{0 l}^{2}(\overrightarrow{\mathrm{n}}) \psi_{00}^{2}(\overrightarrow{\mathrm{n}}) s_{0 l}^{2} s_{00}^{2}, \\
& -\lambda \sum_{\overrightarrow{\mathrm{n}}} \psi_{0 l}^{4}(\overrightarrow{\mathrm{n}}) s_{0 l}^{4},
\end{aligned}
$$

and

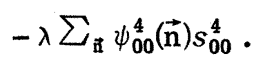

By the normalization condition, $\left|\psi_{00}(\vec{n})\right|$ is of order 1 for $|\vec{n}|$ of order 1; it is zero elsewhere ("elsewhere" being out of the block 0 ). The function $\left|\psi_{0 l}(\overrightarrow{\mathrm{n}})\right|$ is of order $2^{-l d / 2}$ for $|\overrightarrow{\mathrm{n}}| \lesssim 2^{l}$ and zero elsewhere. The sums over $\vec{n}$ are restricted to $|\vec{n}| \sim 1$ when $\psi_{00}(\overrightarrow{\mathrm{n}})$ is involved; the term involving $\psi_{0 t}^{4}(\overrightarrow{\mathrm{n}})$ is summed over all $\vec{n}$ with $|\vec{n}| \leqslant 2^{l}$. With these orders of magnitude the three terms cited above are of order $\lambda 2^{-l d} s_{0 l}^{2} s_{00}^{2}, \lambda 2^{-l d} s_{0 l}^{4}$, and $\lambda s_{00}^{4}$, respectively. To estimate the size of these terms requires an estimate of the size of $s_{00}$ and $s_{0 l}$; such estimates will be made later. But independently of the size of the variables $s_{00}$ and $s_{01}$ the coupling term is smaller than one of the diagonal terms. If $\left|s_{0 l}\right| \gg\left|s_{00}\right|$ then the $s_{0 l}^{4}$ term is much larger than the coupling term, while if $\left|s_{0 i}\right| \lesssim\left|s_{00}\right|$ the $s_{00}^{4}$ term is larger than the coupling term. This conclusion is not necessarily true for $l=1$ because the $s_{00}^{2} s_{0 t}^{2}$ term has an extra factor of 6 in front of it. The effect of the coupling term with $l=1$ will be discussed in Sec. III.

One can now see that the division of phase space is essentially uniquely determined. If one makes any of the $k$-space shells much larger, the $K$-type coupling terms between neighboring position-space blocks become too large. If one tries to make smaller $k$-space shells, i. e., shells whose width $\Delta k$ is much smaller than the mean value of $k$, then one has many shells having about the same volume, so that the corresponding position-space blocks are the same size, and this means large coupling from the $\lambda \sum_{\text {iी }} s_{\mathrm{i}}^{4}$ term. The precise definition of the momentum-space shells ( $k$ changing by a factor 2 in a shell) will be discussed in Sec. III.

\section{Structure of Partition Function: Diagonal Terms}

Now a systematic analysis of the partition function will be described, using the variables $s_{\overrightarrow{\mathrm{m}} l}$ defined above. For simplicity only the case $h=0$ will be discussed. ${ }^{13}$ First the diagonal terms will be examined; later the effect of coupling terms will be discussed.

Let the diagonal terms in the exponential of Eq. (1.2) be denoted $\mathfrak{H}_{0}$. To be explicit, $\mathfrak{H}_{0}$ is

$$
\begin{aligned}
\mathcal{H}_{0}= & \sum_{\overrightarrow{\mathrm{m}}} \sum_{l} \mathcal{H}_{\overrightarrow{\mathrm{m}} l}, \\
\mathcal{H}_{\overrightarrow{\mathrm{m}} l}=-r & \sum_{\overrightarrow{\mathrm{n}}} \psi_{\overrightarrow{\mathrm{m}} l}^{2}(\overrightarrow{\mathrm{n}}) s_{\overrightarrow{\mathrm{m}} l}^{2}-\lambda \sum_{\overrightarrow{\mathrm{n}}}\left[\psi_{\overrightarrow{\mathrm{m}} l}(\overrightarrow{\mathrm{n}})\right]^{4} s_{\overrightarrow{\mathrm{m}} l}^{4} \\
& \quad+K \sum_{\overrightarrow{\mathrm{n}}} \sum_{i} \psi_{\overrightarrow{\mathrm{m}} l}(\overrightarrow{\mathrm{n}}) \psi_{\overrightarrow{\mathrm{m}} l}(\overrightarrow{\mathrm{n}}+\hat{i}) s_{\overrightarrow{\mathrm{m}} l}^{2}
\end{aligned}
$$

The terms proportional to $s_{\mathrm{m} l}^{2}$ are best calculated in momentum space; they have the form $\int_{\overrightarrow{\mathbf{k}}} \phi_{\overrightarrow{\mathrm{m}} l}(\overrightarrow{\mathrm{k}})$ $\times \phi_{\overrightarrow{\mathrm{m}} l}(-\overrightarrow{\mathrm{k}})\left(-r+K \sum_{i} \cos k_{i}\right) s_{\overrightarrow{\mathrm{m}} l}^{2}$, which is roughly

$$
\begin{aligned}
& {\left[(-r+K d) \int_{\overrightarrow{\mathbf{k}}} \phi_{\overrightarrow{\mathrm{m}} l}(\overrightarrow{\mathrm{k}}) \phi_{\overrightarrow{\mathrm{m}} l}(-\overrightarrow{\mathrm{k}})\right.} \\
& \left.\quad-K \int_{\overrightarrow{\mathbf{k}}}\left(k^{2} / 2\right) \phi_{\overrightarrow{\mathrm{m}} l}(\overrightarrow{\mathrm{k}}) \phi_{\overrightarrow{\mathrm{m}} l}(-\overrightarrow{\mathrm{k}})\right] s_{\mathrm{m} l}^{2} .
\end{aligned}
$$


The two terms must be separated because of the possible cancellation of $r$ against $K d$. In the second term $k^{2}$ can be approximated by its order of magnitude in the $l$ th shell, namely, $k^{2} \sim 2^{-2 l}$. The remaining integrals are 1 , by normalization. The coefficient of $s_{\overrightarrow{\mathrm{m}} l}$ can be calculated using orders of magnitude given earlier. One now has an order of magnitude for $\mathfrak{H}_{\overrightarrow{\mathrm{m}} l}$ :

$$
\mathcal{H}_{\overrightarrow{\mathrm{m}} l} \sim\left(-r+K d-2^{-2 l} K\right) s_{\mathrm{m} l}^{2}-\lambda 2^{-d l} s_{\mathrm{m} t}^{4} .
$$

The partition function with coupling terms neglected is, roughly,

$$
\begin{aligned}
Z= & \Pi_{\overrightarrow{\mathrm{m}}} \Pi_{l} \int_{-\infty}^{\infty} d s_{\overrightarrow{\mathrm{m}} l} \\
& \times \exp \left\{\sum_{\overrightarrow{\mathrm{m}}} \Sigma_{l}\left[\left(-r+K d-2^{-2 l} K\right) s_{\mathrm{m} l}^{2}-\lambda 2^{-d l} s_{\overrightarrow{\mathrm{m}} l}^{4}\right]\right\} .
\end{aligned}
$$

It is also interesting to compute the Fourier transform $g(\vec{k})$ of the spin-spin correlation function. The exact definition of $g(\vec{k})$ is ${ }^{14}$ (at or above the critical temperature)

$$
\begin{aligned}
& g(\overrightarrow{\mathrm{k}})=Z^{-1} \prod_{\overrightarrow{\mathbf{n}}} \int d s_{\overrightarrow{\mathbf{n}}} \sigma_{\mathbf{k}} s_{0}
\end{aligned}
$$

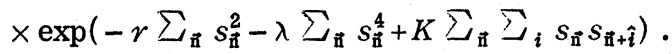

The product $\sigma_{\overrightarrow{\mathbf{k}}} s_{0}$ can be expressed in terms of the variables $s_{\overrightarrow{\mathrm{m}} t}$. Let $\overrightarrow{\mathrm{k}}$ be in the momentum shell $l_{1}$. For any $l$, the lattice site $\overrightarrow{\mathrm{n}}=0$ lies in the positionspace block $\vec{m}=0$. Therefore one has

$$
\sigma_{\overrightarrow{\mathbf{k}}} s_{0}=\sum_{\overrightarrow{\mathrm{m}}} \sum_{l} \phi_{\overrightarrow{\mathrm{m}} l_{1}}(\overrightarrow{\mathrm{k}}) \psi_{0 l}(0) s_{\overrightarrow{\mathrm{m}} l_{1}} s_{0 l} \text {. }
$$

With $Z$ simplified to the form (2.14), the products $s_{\overrightarrow{\mathrm{m}} l_{1}} s_{0 l}$ average to zero if $s_{\overrightarrow{\mathrm{m}} l_{1}}$ is distinct from $s_{0 l}$. Hence one can replace $\sigma_{\overrightarrow{\mathrm{k}}} s_{0}$ by $\phi_{0 l_{1}}(\overrightarrow{\mathrm{k}}) \psi_{0 l_{1}}(0) s_{0 l_{1}}^{2}$; from the orders of magnitude of $\phi_{0 l_{1}}(\overrightarrow{\mathrm{k}})$ and $\psi_{0 l_{1}}(0)$ (see Table I), this is of order $s_{0 t_{1}}^{2}$. Thus the value of $g(\vec{k})$ in order of magnitude is simply the order of magnitude of $s_{0 t_{1}}^{2}$. This can easily be determined from the order-of-magnitude form for $Z$. If $K d<r$ (this will mean $T>T_{c}$ ) then one can neglect the $s_{\mathrm{m} l}^{4}$ term in $Z$, at least for large $l$, and the order of magnitude of $s_{\mathrm{m} l}^{2}$ is $(r-K d)^{-1}$. This means $\left\langle s_{\mathrm{m} l}^{2}\right\rangle$ is constant for large $l$ and $g(\vec{k})$ has no singularity for $\overrightarrow{\mathrm{k}} \rightarrow 0$. If $K d=r$ the exponent in Eq. (2.14) has the form $\sum_{\overrightarrow{\mathrm{m}}} \sum_{l}\left(-K 2^{-2 l} s_{\mathrm{m} l}^{2}-\lambda 2^{-d t} s_{\mathrm{m} l}^{4}\right)$. There is now a competition between the Gaussian and quartic terms to determine which will cut off the exponential and fix the order of magnitude of $s_{\mathrm{m} l}^{2}$. The Gaussian term cuts off the exponential when $s_{\overrightarrow{\mathrm{m}} l}^{2}$ is of order $2^{2 t}$ while the quartic term cuts off the exponential when $s_{\mathrm{m} l}^{2}$ is of order $2^{d l / 2}$. Hence the Gaussian term cuts off first if $d>4$; the quartic term wins if $d<4$, and both terms cut off simultaneously for $d=4$. If $d \geq 4$, one has $\left\langle s_{\mathrm{m} l}^{2}\right\rangle \sim 2^{2 l}$ for large $l$, which means $g(\overrightarrow{\mathbf{k}})$ behaves as $k^{-2}$. This means one is at the critical point; furthermore the behavior $k^{-2}$ is what one expects from mean field theory. For $d<4$ one has $\left\langle s_{\overrightarrow{\mathrm{m}} l}^{2}\right\rangle \sim 2^{d l / 2}$ so that $g(\overrightarrow{\mathrm{k}})$ $\sim k^{-d / 2}$. For $d=3$ this gives $k^{-3 / 2}$; for $d=2$ one gets $k^{-1}$. One is still at the critical point but the exponents do not agree with mean field theory. They do not agree with experiment or numerical solutions of the Ising model either. However, one still has to determine the effects of coupling terms, and these will change the exponents.

\section{Structure of Partition Function: Couplings}

The next step is to study the effects of coupling terms. These are of two types. First there are terms coupling different position-space boxes in the same momentum shell, arising from the $K \sum_{\overrightarrow{\mathbf{l}}} \sum_{i} s_{\overrightarrow{\mathrm{n}}} s_{\overrightarrow{\mathrm{n}}+\hat{i}}$ term. These coupling terms are reduced in size from the original nearest-neighbor coupling strength $K$. But at the critical point the constant $K$ is just large enough so a perturbation expansion in the complete $K$ term diverges. ${ }^{3}$ Since only a part of the $K$ term consists of coupling between different position-space boxes, this coupling should be easily handled by perturbation theory and does not affect the qualitative analysis described earlier. This point will be discussed further later, without changing the conclusion.

The crucial coupling terms are those from the $\lambda \sum_{\overrightarrow{\mathrm{n}}} s_{\mathrm{i}}^{4}$ term. These will be discussed in two stages. First the terms coupling the shell $l=0$ to a particular large $l$ shell will be discussed; afterwards a more general analysis will be presented including the troublesome terms coupling neighboring momentum shells.

To get a preliminary understanding of the effects of coupling terms between different momentum shells, consider the coupling between two specific shells, namely, the $l=0$ shell and a shell with a

TABLE I. Orders of magnitude associated with the phase-space cell $(\overrightarrow{\mathrm{m}}, l)$.

\begin{tabular}{cc}
\hline Quantity & Order of magnitude \\
\hline$\langle\overrightarrow{\mathrm{x}}\rangle$ & $\overrightarrow{\mathrm{m}} a_{l}$ \\
$a_{l}$ & $2^{l} w^{-1 / d}$ \\
$\left\langle k^{2}\right\rangle$ & $\rho_{0} 2^{-2 l}$ \\
$V_{x}$ & $2^{l d} w^{-1}$ \\
$V_{k}$ & $2^{-l d} w$ \\
$\left|\psi_{\overrightarrow{\mathrm{m}} l}(\overrightarrow{\mathrm{x}})\right|^{\mathrm{a}}$ & $2^{-l d / 2} w^{1 / 2}$ \\
$\left|\phi_{\mathrm{m}}(\overrightarrow{\mathrm{k}})\right|^{\mathrm{b}}$ & $2^{l d / 2} w^{-1 / 2}$ \\
$\left\langle s_{\mathrm{m} l}^{2}\right\rangle^{c}$ & $2^{2 l}$ or $2^{\mathrm{d} d / 4}$ \\
$w$ & of order 1 \\
\hline \hline
\end{tabular}

${ }^{\mathrm{a}}$ For $\overrightarrow{\mathrm{x}}$ in box $\overrightarrow{\mathrm{m}}$, i.e., for $\left|\overrightarrow{\mathrm{x}}-\overrightarrow{\mathrm{m}} a_{l}\right|$ of order $a_{l}$.

${ }^{b_{F}}$ or $\overrightarrow{\mathrm{k}}$ in shell $l$, i.e., $2^{-l}<|\overrightarrow{\mathrm{k}}|<2 \times 2^{-l}$.

cAt the critical point.

${ }^{d}$ The estimate $2^{l d / 4}$ is obtained in Sec. II for dimension $d<4$; a better estimate (obtained later in Sec. III) gives $2^{2 l}$ always. 
large but fixed value of $l$. All other shells will be ignored in this preliminary analysis. Furthermore, only the coupling terms proportional to $s_{\overrightarrow{\mathrm{m}} 0}^{2} s_{\mathrm{m}_{1} l}$ will be considered ( $\overrightarrow{\mathrm{m}}$ and $\overrightarrow{\mathrm{m}}_{1}$ are arbitrary). In principle there are other coupling terms, for example, terms proportional to $s_{\mathrm{m} 0}^{\frac{3}{3}} s_{\overrightarrow{\mathrm{m}}_{1} l}$, but these will be dropped in the preliminary analysis.

For $s_{\mathrm{m} 0}^{2} s_{\mathrm{m}_{1} l}^{2}$ to occur as a coupling term with nonzero coefficient, the position-space boxes labeled by $\overrightarrow{\mathrm{m}}$ and $\overrightarrow{\mathrm{m}}_{1}$ must overlap. The boxes associated with shell $l$ are much larger than the boxes associated with shell 0 . Call the boxes associated with shell $l$ "large boxes," and the boxes associated with shell 0 "small boxes." The large boxes have a volume of order $2^{l d}$, the small boxes a volume of order 1. A single large box, such as the box $\overrightarrow{\mathrm{m}}_{1}$, overlaps with $2^{\text {ld }}$ small boxes. A single small box, such as the box $\vec{m}$, overlaps only one large box. Let the large box $\overrightarrow{\mathrm{m}}_{1}$ which overlaps a given small box $\vec{m}$ be the box $\vec{m}_{1}(\vec{m})$. The overlap condition is, roughly,

$$
\left|\overrightarrow{\mathrm{m}}-2^{l} \overrightarrow{\mathrm{m}}_{1}\right|<2^{l} \text {. }
$$

If this condition is satisfied, then the interaction includes a term $-\lambda 2^{-l d} s{ }_{\overrightarrow{\mathrm{m}} 0}^{2} s_{\overrightarrow{\mathrm{m}}_{1} l}^{2}$ (as calculated earlier). Consider now the part of the partition function involving the two momentum shells 0 and $l$, and consider only the terms discussed so far. The result is

$$
\begin{aligned}
Z= & \Pi_{\overrightarrow{\mathrm{m}}} \Pi_{\overrightarrow{\mathrm{m}}_{1}} \int_{-\infty}^{\infty} d s_{\overrightarrow{\mathrm{m}} 0} \int_{-\infty}^{\infty} d s_{\overrightarrow{\mathrm{m}}_{1} l} \\
& \times \exp \left\{\sum_{\overrightarrow{\mathrm{m}}}\left[(-r+K d-K) s_{\overrightarrow{\mathrm{m}} 0}^{2}-\lambda s_{\overrightarrow{\mathrm{m}} 0}^{4}\right]\right. \\
& +\sum_{\overrightarrow{\mathrm{m}}_{1}}\left[\left(-r+K d-2^{-2 l} K\right) s_{\mathrm{m}_{1} l}^{2}-2^{-l d} \lambda_{\mathrm{m}_{1} l}^{4}\right] \\
& \left.-\left.\lambda 2^{-l d} \sum_{\overrightarrow{\mathrm{m}}} s_{\mathrm{m}_{0} 0}^{2} s_{\overrightarrow{\mathrm{m}}_{1} l}^{2}\right|_{\overrightarrow{\mathrm{m}}_{1}=\overrightarrow{\mathrm{m}}_{1}(\overrightarrow{\mathrm{m}})} ^{2}\right\} .
\end{aligned}
$$

Using the estimated orders of magnitude for $s_{\overrightarrow{\mathrm{m}} 0}$ and $s_{\overrightarrow{\mathrm{m}}_{1} l}$ obtained earlier, the product $s_{\overrightarrow{\mathrm{m}} 0}^{2} s_{\overrightarrow{\mathrm{m}}_{1} l}^{2}$ is at most of order $2^{l d / 2}$ (using the estimate $2^{l d / 4}$ for $s \overrightarrow{\mathrm{m}}_{1} l$ ). In five dimensions or higher this is an overestimate. This means $2^{-l d} s_{\overrightarrow{\mathrm{m}} 0}^{2} s_{\overrightarrow{\mathrm{m}}_{1} l}^{2}$ is at most of or$\operatorname{der} 2^{-l d / 2}$ which is small. This means that the coupling term involving any given spin $s_{\overrightarrow{\mathrm{m}} 0}$ from a small box is small compared to the diagonal term involving $s_{\overrightarrow{\mathrm{m}} 0}$. The situation is different if one compares coupling terms and diagonal terms involving a given spin $s_{\overrightarrow{\mathrm{m}}_{1} l}$ from a large box. The trouble is that the spin $s_{\overrightarrow{\mathrm{m}}_{1} l}$ appears in $2^{l d}$ coupling terms, since $2^{l} d$ small boxes fit into one large box. The sum total of these coupling terms (all of which have the same sign) is of order $2^{l d / 2}$ (for $d \leq 4$ ). This is much larger than the diagonal term involving $s_{\vec{m}_{1} l}$.

Since the coupling terms are small compared to the diagonal terms from shell 0 , it is reasonable to do the integrations over $s_{\overrightarrow{\mathrm{m}} 0}$ treating the coupling terms as a perturbation. The $s_{\overrightarrow{\mathrm{m}}_{1} l}$ integrations will be postponed until later. The form of an $s_{\overrightarrow{\mathrm{m}} 0}$ integration is

$$
I_{\overrightarrow{\mathrm{m}}}=\int_{-\infty}^{\infty} d s_{\overrightarrow{\mathrm{m}} 0} \exp \left[(-r+K d-K) s_{\overrightarrow{\mathrm{m}} 0}^{2}-\lambda s_{\overrightarrow{\mathrm{m}} 0}^{4}-x_{\overrightarrow{\mathrm{m}}} s_{\overrightarrow{\mathrm{m}} 0}^{2}\right],
$$

where

$$
x_{\overrightarrow{\mathrm{m}}}=\left.\lambda 2^{-l d} s_{\overrightarrow{\mathrm{m}}_{1} l}^{2}\right|_{\overrightarrow{\mathrm{m}}_{1}=\overrightarrow{\mathrm{m}}_{1}(\overrightarrow{\mathrm{m}})} \text {. }
$$

Because $x_{\overrightarrow{\mathrm{m}}}$ is small, one expands the integral $I_{\overrightarrow{\mathrm{m}}}$ in powers of $x \overrightarrow{\mathrm{m}}$. The expansion has the form

$$
I_{\overrightarrow{\mathrm{m}}}=c_{0}+c_{1} x_{\overrightarrow{\mathrm{m}}}+c_{2} x_{\overrightarrow{\mathrm{m}}}^{2}+\cdots,
$$

where $c_{0}, c_{1}$, etc. are constants of order $1\left(c_{0}, c_{1}\right.$, etc. will be functions of $r, K$, and $\lambda$ but not of $l$ ). It is convenient to write $I_{\vec{m}}$ in exponential form; it still has a power series expansion, say,

$$
I_{\overrightarrow{\mathrm{m}}}=\exp \left(b_{0}+b_{1} x_{\overrightarrow{\mathrm{m}}}+b_{2} x_{\overrightarrow{\mathrm{m}}}^{2}+\cdots\right),
$$

with $b_{0}=\ln c_{0}, b_{1}=c_{1} / c_{0}$, etc. The partition function now has the form (still keeping only the two momentum shells)

$$
\begin{aligned}
Z= & \Pi_{\overrightarrow{\mathrm{m}}_{1}} \int_{-\infty}^{\infty} d s_{\overrightarrow{\mathrm{m}}_{1} l}\left(\Pi_{\overrightarrow{\mathrm{m}}} I_{\overrightarrow{\mathrm{m}}}\right) \\
& \quad \times \exp \left\{\sum_{\overrightarrow{\mathrm{m}}_{1} l}\left[\left(-r+K d-2^{-2 l} K\right) s_{\overrightarrow{\mathrm{m}}_{1} l}^{2}-2^{-l d} \lambda s_{\overrightarrow{\mathrm{m}}_{1} l}^{4}\right]\right\} \\
= & \Pi_{\overrightarrow{\mathrm{m}}_{1}} \int_{-\infty}^{\infty} d s_{\overrightarrow{\mathrm{m}}_{1} l} \\
& \quad \times \exp \left\{\sum _ { \vec { \mathrm { m } } _ { 1 } } \left[2^{l d} b_{0}+\left(-r+K d-2^{-2 l} K+\lambda b_{1}\right) s_{\overrightarrow{\mathrm{m}}_{1} l}^{2}\right.\right. \\
& \left.\left.\quad-2^{-l d}\left(\lambda-\lambda^{2} b_{2}\right) s_{\overrightarrow{\mathrm{m}}_{1} l}+\lambda^{3} 2^{-2 l d} b_{3} s_{\overrightarrow{\mathrm{m}}_{1} l}^{6}-\cdots\right]\right\} .
\end{aligned}
$$

In the last expression, terms from the product $\Pi_{\overrightarrow{\mathrm{m}}} I_{\overrightarrow{\mathrm{m}}}$ have been converted to sums over $\overrightarrow{\mathrm{m}}_{1}$ in the exponent; a factor $2^{l d}$ is included because there are $2^{l d}$ boxes $\overrightarrow{\mathrm{m}}$ for each box $\overrightarrow{\mathrm{m}}_{1}$.

One now has the partition function expressed as an integral only over $l$-shell variables $s_{\overrightarrow{\mathrm{m}}_{1} l}$. The effect of the coupling terms is to make nontrivial changes in the coefficients of $s_{\overrightarrow{\mathrm{m}}_{1} l}^{2}, s_{\mathrm{m}_{1} l}^{4}$, etc. As a result one has a new condition for being at the critical temperature; one must have

$$
-r+K d-\lambda b_{1}=0
$$

to ensure that the order of magnitude of $s_{\mathrm{m}_{1} l}^{2}$ goes to infinity as $l \rightarrow \infty$. As long as this condition is satisfied, the coefficients of $s_{\mathrm{m}_{1} l}^{2}$ and $s_{\mathrm{m}_{1} l}^{4}$ have the same order of magnitude as when the coupling terms were neglected. It is easily seen that the $s_{\overrightarrow{\mathrm{m}}_{1} l}^{6}$ terms and higher are negligible.

If one only had to discuss coupling between an arbitrary shell $l$ with large $l$ and the shell 0 , this would end the discussion. However in practice one has couplings between all pairs of shells. But one can imagine what the effects will be of these couplings. Suppose one can start by integrating out 
the variables $s_{\overrightarrow{\mathrm{m}} 0}$ treating the coupling to other shells as a perturbation. The result will be to get a new form for $Z$ involving only $s_{\overrightarrow{\mathrm{m}} 1}, s_{\overrightarrow{\mathrm{m}} 2}$, etc. The effective interaction between these spins will involve new coefficients for the $s_{\overrightarrow{\mathrm{m}} l}^{2}$ and $s_{\overrightarrow{\mathrm{m}} l}$ terms. Then one integrates the spins $s_{\overrightarrow{\mathrm{m}} 1}$ from the shell $l=1$, treating the coupling of this shell to remaining shells as a perturbation. The result of this is to produce a second effective interaction involving the spins $s_{\overrightarrow{\mathrm{m}} 2}, s_{\overrightarrow{\mathrm{m}} 3}$, etc. To compute the partition function one repeats this process an infinite number of times, until all spins have been integrated.

When one carries through this sequence of integrations taking into account couplings between all pairs of momentum shells, one gets results qualitatively different from those obtained keeping only diagonal terms. The reason is the following. In the analysis keeping only diagonal terms, the coef-

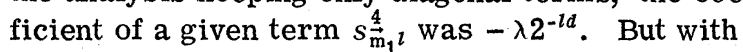
off-diagonal terms taken into account, the coefficient of $s_{\mathrm{m}_{1} l}^{4}$ changes each time a momentum shell is integrated out. As a result the constant $\lambda$ is replaced by a succession of constants $\lambda_{1}, \lambda_{2}, \ldots, \lambda_{l-1}$ which only stops when $s_{\overrightarrow{\mathrm{m}}_{1} l}$ itself is integrated out. From the previous analysis one sees that the difference $\lambda_{k}-\lambda_{k-1}$ is nontrivial for any $k$ between 1 and $l-1$; as a result $\lambda_{l}$ can have a strong $l$ dependence; $\lambda_{l} 2^{-l d}$ may be very different from $\chi^{-l d}$, which would change the order of magnitude of $s_{m l}$ from previous estimates.

Another problem arises when all couplings between momentum shells are taken into account. The couplings between neighboring momentum shells are not small and cannot be treated in perturbation theory. For example, if the specific coupling discussed previously were the coupling to the shell $l=1$, the quantities $x_{\overrightarrow{\mathrm{m}}}$ are not necessarily small, and in addition there is a factor of 6 in the coupling term. Hence the integrals $I_{\overrightarrow{\mathrm{m}}}$ probably cannot be expanded in powers of $x \overrightarrow{\mathrm{m}}_{\mathrm{m}}$. As a result the effective interaction one gets after integrating out one or more momentum shells will be more complicated, involving complicated functions of $s_{\overrightarrow{\mathrm{m}} l}$ rather than just quadratic and quartic terms.

\section{IMPROVED ANALYSIS OF PARTITION FUNCTION}

\section{A. Continuum Approximation}

Now a more careful analysis of the partition function will be carried out taking into account all the couplings between momentum shells coming from the $\sum_{\vec{n}} s_{\vec{n}}^{4}$ term. The couplings between different spins of the same shell will be ignored; by the argument presented earlier these couplings should be a perturbation and not important in a qualitative analysis. In the following analysis we shall try to estimate all terms to within a factor of 2 or so. As a preliminary we: shall make some technical simplifications in the formulation of the partition function and estimate more carefully some orders of magnitude connected with the division of phase space into cells.

It is convenient to replace the discrete spin variables $s_{\vec{i}}$ by a field $s(\overrightarrow{\mathrm{x}})$ depending on a continuous variable $\vec{x}$. This is accomplished by introducing wave functions $\psi_{\overrightarrow{\mathrm{m}} l}(\overrightarrow{\mathrm{x}})$ depending on $\overrightarrow{\mathrm{x}}$ in place of wave functions depending on $\vec{n}$. Then

$$
s(\overrightarrow{\mathrm{x}})=\sum_{\overrightarrow{\mathrm{m}}} \sum_{l=0}^{\infty} \psi_{\overrightarrow{\mathrm{m}} l}(\overrightarrow{\mathrm{x}}) s_{\overrightarrow{\mathrm{m}} l} .
$$

The wave functions satisfy the orthonormality condition

$$
\int_{\overrightarrow{\mathrm{x}}} \psi_{\mathrm{\textrm {m }} l}^{*}(\overrightarrow{\mathrm{x}}) \psi_{\overrightarrow{\mathrm{m}}_{1} l_{1}}(\overrightarrow{\mathrm{x}})=\delta_{\overrightarrow{\mathrm{m}} \overrightarrow{\mathrm{m}}_{1}} \delta_{l l_{1}},
$$

where $\int_{\overrightarrow{\mathbf{x}}}$ means $\int d^{d} x$. Replacing $s_{\overrightarrow{\mathrm{n}}}$ by $s(\overrightarrow{\mathrm{x}})$ is a minor change. For large $l$ one cannot distinguish $\psi_{\overrightarrow{\mathrm{m}} l}(\overrightarrow{\mathrm{n}})$ from $\psi_{\overrightarrow{\mathrm{m}} l}(\overrightarrow{\mathrm{x}})$ since $\psi_{\overrightarrow{\mathrm{m}} l}(\overrightarrow{\mathrm{n}})$ should be slowly varying over distances of order 1. For small $l$, for which a position-space box covers only a few lattice sites, the functions $\psi_{\overrightarrow{\mathrm{m}} l}(x)$ will differ quantitatively from $\psi_{\overrightarrow{\mathrm{m}} l}(\overrightarrow{\mathrm{n}})$ but the qualitative analysis of this paper is unaffected by the change. The Fourier-transform functions are

$$
\phi_{\overrightarrow{\mathrm{m}} l}(\overrightarrow{\mathrm{k}})=\int_{\overrightarrow{\mathbf{x}}} e^{i \overrightarrow{\mathbf{k}} \cdot \overrightarrow{\mathbf{x}}} \psi_{\overrightarrow{\mathbf{m}} l}(\overrightarrow{\mathbf{x}})
$$

We suppose that the absolute value $\left|\phi_{\overrightarrow{\mathrm{m}} l}(\overrightarrow{\mathrm{k}})\right|$ is to a zeroth approximation constant in the shell $2^{-l}$ $\leq|\overrightarrow{\mathrm{k}}| \leq 2.2^{-t}$ and 0 outside this shell. The normalization condition for $\phi_{\overrightarrow{\mathrm{m}} l}(\overrightarrow{\mathrm{k}})$ is

$$
\int_{\overrightarrow{\mathbf{k}}}\left|\phi_{\overrightarrow{\mathrm{m}} l}(k)\right|^{2}=1 \text {, }
$$

where $\int_{\overrightarrow{\mathbf{x}}}$ means $(2 \pi)^{-d} \int d^{d} k$. The volume of the $l$ th shell is $(2 \pi)^{d} 2^{-l d} w$, where $w$ is a constant; hence from Eq. (3.4) one has

$$
\left|\phi_{\overrightarrow{\mathrm{m}} l}(\overrightarrow{\mathrm{k}})\right| \simeq 2^{l d / 2} w^{-1 / 2} \text {. }
$$

The precise phase-space volume for a degree of freedom is ${ }^{15}(2 \pi)^{d}$; hence the position-space boxes associated with momentum shell $l$ have volume $2^{l d} w^{-1}$. This means the spacing of the positionspace boxes is $a_{l}$, with

$$
a_{t}=2^{l} a_{0},
$$

where

$$
a_{0}=w^{-1 / d} \text {. }
$$

To a zeroth approximation we imagine that $\left|\psi_{\overrightarrow{\mathrm{m}} l}(\overrightarrow{\mathrm{x}})\right|$ is constant inside the box $\overrightarrow{\mathrm{m}}$ and zero outside it; then the normalization condition gives

$$
\left|\psi_{\overrightarrow{\mathrm{m}} l}(\overrightarrow{\mathrm{x}})\right| \simeq 2^{-l d / 2} w^{1 / 2} \text {. }
$$

The quantity $\left|\phi_{\overrightarrow{\mathrm{m}} l}(\overrightarrow{\mathrm{k}})\right|$ for $\overrightarrow{\mathrm{k}}=0$ must be 0 since $\overrightarrow{\mathrm{k}}=0$ is outside the shell $l$. This means $\int_{\overrightarrow{\mathbf{x}}} \psi_{\overrightarrow{\mathrm{m}} l}(\overrightarrow{\mathrm{x}})$ must vanish; this means in turn that $\psi_{\overrightarrow{\mathrm{m}} l}(\overrightarrow{\mathrm{x}})$ must be negative over about half the volume of the box $\vec{m}$. Our picture will be that $\psi_{\overrightarrow{\mathrm{m}} l}(\overrightarrow{\mathrm{x}})=2^{-l d / 2} w^{-1 / 2}$ in half the 
volume and $-2^{l d / 2} w^{-1 / 2}$ in the other half. Quantitatively this picture is absurd, but for qualitative purposes, in particular for integrals involving $\psi_{\overrightarrow{\mathrm{m}} l}(\overrightarrow{\mathbf{x}})$, one hopes that this discontinuous formula is a rough approximation to the correct continuous function.

Any two phase-space cells with different $\vec{m}$ and $l$ can be transformed into each other by combining a translation in position space with scale transformations in position and momentum space. We assume an analogous transformation applies to the wave functions $\psi_{\overrightarrow{\mathrm{m}} l}(\overrightarrow{\mathrm{x}})$, namely,

$$
\psi_{\overrightarrow{\mathrm{m}} l}(\overrightarrow{\mathrm{x}})=\psi_{00}\left(2^{-l} \overrightarrow{\mathrm{x}}-\overrightarrow{\mathrm{m}} a_{0}\right) .
$$

Consider a partitition function of the following form:

$$
Z=\underset{\overrightarrow{\mathrm{m}}}{\prod_{l=0}^{\infty}} \int_{-\infty}^{\infty} d s_{\overrightarrow{\mathrm{m}} l} e^{3 c_{0}},
$$

where

$$
\mathfrak{H}_{0}=-\int_{\overrightarrow{\mathrm{x}}} P[s(\overrightarrow{\mathrm{x}})]-\frac{1}{2} K \int_{\overrightarrow{\mathrm{x}}} \nabla s(\overrightarrow{\mathrm{x}}) \cdot \nabla s(\overrightarrow{\mathrm{x}}) .
$$

The function $P(s)$ will be permitted to be an arbitrary even function of $s$, provided it goes to infinity when $s \rightarrow \infty$ (this is necessary to ensure convergent integrals). The expression $\int_{\overrightarrow{\mathrm{x}}} \nabla s(\overrightarrow{\mathrm{x}}) \cdot \nabla s(\overrightarrow{\mathrm{x}})$ is the continuum form of $2 \sum_{\overrightarrow{\mathrm{n}}} \sum_{i}\left(-s_{\overrightarrow{\mathrm{n}}} s_{\overrightarrow{\mathrm{n}}+\hat{i}}+s_{\overrightarrow{\mathrm{n}}}^{2}\right)$. [When these expressions are converted into momentum space the continuum term is $\int_{\vec{k}} k^{2} \sigma_{\vec{k}} \sigma_{-\vec{k}}$ while the lattice sum becomes $\int_{\vec{k}} \sum_{i}\left(2-2 \cos k_{i}\right) \sigma_{\vec{k}} \sigma_{-\vec{k}}$ which is of order $\int_{\overrightarrow{\mathrm{k}}} k^{2} \sigma_{\overrightarrow{\mathrm{k}}} \sigma_{-\overrightarrow{\mathrm{k}}}$, especially for the small- $\overrightarrow{\mathrm{k}}$ part of the integral.] When the model of Eq. (1.2) is converted to continuum form, one obtains an interaction of the form (3.11) with

$$
P(s)=(r-K d) s^{2}+\lambda s^{4} .
$$

Interactions of the form (3.11) have been discussed by Landau and Ginsberg. ${ }^{5}$

\section{B. Integration Over Shell $l=0$}

One now wants to compute the integrals for degrees of freedom from the shell $l=0$, that is, one wants to integrate the variables $s_{\overrightarrow{\mathrm{m}} 0}$, leaving the variables $s_{\overrightarrow{\mathrm{m}} 1}, s_{\overrightarrow{\mathrm{m}} 2}$, etc. unintegrated. It is convenient to isolate the $s_{\overrightarrow{\mathrm{m}} 0}$ terms in $s(\overrightarrow{\mathrm{x}})$ by writing

$$
s(\overrightarrow{\mathrm{x}})=\sum_{\overrightarrow{\mathrm{m}}} \psi_{\overrightarrow{\mathrm{m}} 0}(\overrightarrow{\mathrm{x}}) s_{\overrightarrow{\mathrm{m}} 0}+s_{1}(\overrightarrow{\mathrm{x}}),
$$

where

$$
s_{1}(\overrightarrow{\mathrm{x}})=\sum_{\overrightarrow{\mathrm{m}}} \sum_{l=1}^{\infty} \psi_{\overrightarrow{\mathrm{m}} l}(\overrightarrow{\mathrm{x}}) s_{\overrightarrow{\mathrm{m}} l} .
$$

The result of integrating the variables $s_{\overrightarrow{\mathrm{m}} 0}$ will be to express $Z$ as an integral over the variables $s_{\overrightarrow{\mathrm{m}} 1}, s_{\overrightarrow{\mathrm{m}} 2}$, etc., with an effective interaction $\mathfrak{H}_{1}$ depending on the field $s_{1}(\overrightarrow{\mathrm{x}})$. Then one has to set up the problem of integrating the variables $s_{\overrightarrow{\mathrm{m}} 1}$; when these integrations have been carried out one must integrate the variables $s_{\overrightarrow{\mathrm{m}} 2}$, and so on. It is con- venient to make a scale transformation such that $\mathfrak{H}_{1}$ is an interaction of exactly the same form as $\mathfrak{H}_{0}$, so that the analysis of $\mathfrak{H}_{0}$ can be used to integrate $\mathfrak{H}_{1}$ too. The scale transformation is obtained as follows. From the scaling law for the wave functions, one has

$$
\psi_{\overrightarrow{\mathrm{m}} l}(\overrightarrow{\mathrm{x}})=2^{-d / 2} \psi_{\overrightarrow{\mathrm{m}} l-1}(\overrightarrow{\mathrm{x}} / 2) .
$$

If one writes

$$
s_{1}(\overrightarrow{\mathrm{x}})=2^{-d / 2} \alpha s^{\prime}(\overrightarrow{\mathrm{x}} / 2)
$$

( $\alpha$ is a constant that will be fixed later) then

$$
s^{\prime}(\overrightarrow{\mathrm{x}})=\alpha^{-1} \sum_{\overrightarrow{\mathrm{m}}} \sum_{l=1}^{\infty} \psi_{\overrightarrow{\mathrm{m}} l-1}(\overrightarrow{\mathrm{x}}) s_{\overrightarrow{\mathrm{m}} l} .
$$

If one defines

$$
s_{\overrightarrow{\mathrm{m}} l-1}^{\prime}=\alpha^{-1} s_{\overrightarrow{\mathrm{m}} l}
$$

one has

$$
s^{\prime}(\overrightarrow{\mathrm{x}})=\sum_{\overrightarrow{\mathrm{m}}} \sum_{l=0}^{\infty} \psi_{\overrightarrow{\mathrm{m}} l}(\overrightarrow{\mathrm{x}}) s_{\mathrm{m} l}^{\prime} .
$$

Since the sum over $l$ now starts at zero, $s^{\prime}(\overrightarrow{\mathrm{x}})$ is a field of the same form as the original field $s(\overrightarrow{\mathrm{x}})$. $\mathcal{H}_{1}$ will be expressed in terms of $s^{\prime}(\overrightarrow{\mathrm{x}})$ instead of $s_{1}(\overrightarrow{\mathrm{x}})$.

The constant $\alpha$ is an arbitrary scale factor in the definition of $s^{\prime}(\overrightarrow{\mathrm{x}})$. It will ultimately be chosen to make the coefficient of the $\int \nabla s^{\prime}(\overrightarrow{\mathrm{x}})^{2}$ term in $\mathcal{F}_{1}$ be the same as the coefficient $K$ in $\mathcal{H}$. For now it will be left as an arbitrary constant.

Substituting $s^{\prime}$ for $s_{1}$, one has

$$
s(\overrightarrow{\mathrm{x}})=\sum_{\overrightarrow{\mathrm{m}}} \psi_{\overrightarrow{\mathrm{m}} 0}(\overrightarrow{\mathrm{x}}) s_{\overrightarrow{\mathrm{m}} 0}+2^{-d / 2} \alpha s^{\prime}(\overrightarrow{\mathrm{x}} / 2) .
$$

Now one can write $\mathfrak{H}_{0}$ in terms of $s_{\overrightarrow{\mathrm{m}} 0}$ and $s^{\prime}(\overrightarrow{\mathrm{x}})$. The gradient term becomes

$$
\begin{aligned}
\int_{\overrightarrow{\mathrm{x}}} & \nabla s(\overrightarrow{\mathrm{x}}) \cdot \nabla s(\overrightarrow{\mathrm{x}}) \\
& \simeq \rho_{0} \sum_{\overrightarrow{\mathrm{m}}} s_{\overrightarrow{\mathrm{m}} 0}^{2}+\alpha^{2} 2^{-d} \int_{\overrightarrow{\mathrm{x}}} \nabla\left[s^{\prime}(\overrightarrow{\mathrm{x}} / 2)\right] \cdot \nabla\left[s^{\prime}(\overrightarrow{\mathrm{x}} / 2)\right], \\
\rho_{0} & =\int_{\overrightarrow{\mathrm{x}}}\left[\nabla \psi_{\overrightarrow{\mathrm{m}} 0}(\overrightarrow{\mathrm{x}})\right]^{2}
\end{aligned}
$$

[where this integral is independent of $\overrightarrow{\mathrm{m}}$, owing to Eq. (3.9)]. In Eq. (3.21) coupling terms (proportional to $s_{\overrightarrow{\mathrm{m}} 0} s_{\overrightarrow{\mathrm{m}}_{1} 0}$ with $\overrightarrow{\mathrm{m}}_{1} \neq \overrightarrow{\mathrm{m}}$ ) have been neglected. There are no cross terms proportional to $s_{\overrightarrow{\mathrm{m}} 0} s^{\prime}(\overrightarrow{\mathrm{x}} / 2)$ because the gradient term does not couple degrees of freedom from different momentum shells [cf. Eq. (2.2)]. The size of the coupling terms relative to the size of the term $\sum_{\overrightarrow{\mathrm{m}}} s_{\overrightarrow{\mathrm{m}} 0}^{2}$ is determined by the ratio of the variation of $k^{2}$ within the shell $l=0$ to the mean value of $k^{2}$ in the shell. The reason for this is that $\rho_{0}$ is equal to the average value of $k^{2}$ within the shell $l=0$ from Eq. (3.22), while the coefficient of $s_{\overrightarrow{\mathrm{m}} 0} s_{\overrightarrow{\mathrm{m}}_{10} 0}$ can be written $\int_{\overrightarrow{\mathbf{k}}}\left(k^{2}-\rho_{0}\right)$ $\times \phi_{\mathrm{m} 0}^{*}(\overrightarrow{\mathrm{k}}) \phi_{\overrightarrow{\mathrm{m}}_{1} 0}(\overrightarrow{\mathrm{k}})$ (the subtraction of $\rho_{0}$ does not change the integral because the wave functions are ortho- 
gonal). The integral of the absolute value of the wave functions by themselves is 1 , from their order of magnitude, so the full integral cannot exceed the maximum value of $\left|k^{2}-\rho_{0}\right| ;$ i.e., the integral is a measure of the fluctuation of $k^{2}$ within the shell from its average value $\rho_{0}$. This fluctuation should be smaller, by a factor of 2 or 3 , than $\rho_{0}$ itself. In any case, the fluctuation of $k^{2}$ within the shell $l=0$ is smaller by a factor of 2 or so than the fluctuation of $k^{2}$ if one kept the full range of $k$ in each phase-space cell. But keeping the full range of $k$ in each phase-space cell is equivalent to using the original lattice variables; in terms of these variables the nearest-neighbor coupling terms are just large enough so that a perturbation expansion in the coupling terms diverges. ${ }^{3}$ By considering only the shell $l=0$, the size of the coupling term is reduced by a factor of 2 or so from the critical size, and will be neglected.

The $P$ term in $\mathcal{H}_{0}$ is calculated as follows. First one breaks up the integration over all $\vec{x}$ into integrations over each position-space box $\overrightarrow{\mathrm{m}}$. Within the box $\overrightarrow{\mathrm{m}}$ one has

$$
s(\overrightarrow{\mathrm{x}})=\psi_{\overrightarrow{\mathrm{m}} 0}(\overrightarrow{\mathrm{x}}) s_{\overrightarrow{\mathrm{m}} 0}+2^{-d / 2} \alpha s^{\prime}(\overrightarrow{\mathrm{x}} / 2)
$$

so the integral over the box $\vec{m}$ is

$$
\int_{\overrightarrow{\mathbf{x}} \in \text { box } \overrightarrow{\mathrm{m}}} P\left[\psi_{\overrightarrow{\mathrm{m}} 0}(\overrightarrow{\mathrm{x}}) s_{\overrightarrow{\mathrm{m}} 0}+2^{-d / 2} \alpha s^{\prime}(\overrightarrow{\mathrm{x}} / 2)\right] .
$$

Now the degrees of freedom included in $s^{\prime}(\overrightarrow{\mathrm{x}} / 2)$ are associated with position-space boxes which are larger than box $\vec{m}$ by at least a factor of 2 . This suggests that $s^{\prime}(\overrightarrow{\mathrm{x}} / 2)$ will be essentially constant as $\overrightarrow{\mathrm{x}}$ varies over the box $\overrightarrow{\mathrm{m}}$. So approximate $s^{\prime}(\overrightarrow{\mathrm{x}} / 2)$ by its value at some point inside the box, for example, the point $x=\overrightarrow{\mathrm{m}} a_{0}$. Now since $\psi_{\overrightarrow{\mathrm{m}} 0}(\overrightarrow{\mathrm{x}})$ is negative (namely, $-w^{1 / 2}$ ) over half the box $\overrightarrow{\mathrm{m}}$ and positive over the other half, one has

$$
\begin{aligned}
\int_{\overrightarrow{\mathrm{x}}} P[s(\overrightarrow{\mathrm{x}})] \simeq & \sum_{\overrightarrow{\mathrm{m}}} \frac{1}{2} w^{-1}\left\{P\left[-w^{1 / 2} s_{\overrightarrow{\mathrm{m}} 0}+2^{-d / 2} \alpha s^{\prime}\left(\overrightarrow{\mathrm{m}} a_{0} / 2\right)\right]\right. \\
& \left.+P\left[w^{1 / 2} s_{\overrightarrow{\mathrm{m}} 0}+2^{-d / 2} \alpha s^{\prime}\left(\overrightarrow{\mathrm{m}} a_{0} / 2\right)\right]\right\}
\end{aligned}
$$

The factor $\frac{1}{2} w^{-1}$ is half the volume of box $\vec{m}$.

The replacement of $s^{\prime}(\overrightarrow{\mathrm{x}} / 2)$ by a constant within the cell $\overrightarrow{\mathrm{m}}$ is the second major approximation made in the qualitative analysis of the partition function. The basic idea behind this approximation is that since $s^{\prime}(\overrightarrow{\mathrm{x}} / 2)$ only contains momenta $k<0.5$, it should be more slowly varying than the wave function $\psi_{\overrightarrow{\mathrm{m}} 0}(\overrightarrow{\mathrm{x}})$ which contains only momenta $0.5<k<1$. Roughly speaking the wavelengths of $s^{\prime}(\overrightarrow{\mathrm{x}} / 2)$ should be 2 or 3 times the wavelength of $\psi_{\overrightarrow{\mathrm{m}} 0}(\overrightarrow{\mathrm{x}})$. In the phase-space cell $\overrightarrow{\mathrm{m}}, \psi_{\overrightarrow{\mathrm{m}} \mathbf{0}}(\overrightarrow{\mathrm{x}})$ goes through essentially one complete cycle, in our crude picture. So $s^{\prime}(\overrightarrow{\mathrm{x}} / 2)$ should go through only a fraction of a cycle; the simplest approximation one can make to $s^{\prime}(\overrightarrow{\mathrm{x}} / 2)$ is to replace it by a constant. It is hard to evaluate how good an approximation this is.
The approximation of replacing $s^{\prime}(\overrightarrow{\mathrm{x}} / 2)$ by a constant is sufficient to make practical the calculation of the integrals over $s_{\overrightarrow{\mathrm{m}} 0}$. This means one does not have to assume that the coupling between $s_{\overrightarrow{\mathrm{m}} 0}$ and the variables in $s^{\prime}(\overrightarrow{\mathrm{x}} / 2)$ is small. This is fortunate, since it was shown in Sec. II that the coupling between neighboring shells was not likely to be small. This was due especially to the factor of 6 in the coupling term (see Sec. II B).

The form of $\mathcal{H}_{0}$ is now

$$
\begin{aligned}
\mathcal{H}_{0}= & -\frac{1}{2} w^{-1} \sum_{\overrightarrow{\mathrm{m}}}\left\{P\left[-w^{1 / 2} s_{\overrightarrow{\mathrm{m}} 0}+2^{-d / 2} \alpha s^{\prime}\left(\overrightarrow{\mathrm{m}} a_{0} / 2\right)\right]\right. \\
& \left.+P\left[w^{1 / 2} s_{\overrightarrow{\mathrm{m}} 0}+2^{-d / 2} \alpha s^{\prime}\left(\overrightarrow{\mathrm{m}} a_{0} / 2\right)\right]\right\}-\frac{1}{2} K \rho_{0} \sum_{\overrightarrow{\mathrm{m}}} s_{\overrightarrow{\mathrm{m}} 0}^{2} \\
& -\left(K \alpha^{2} / 8\right) \int_{\overrightarrow{\mathrm{x}}} \nabla s^{\prime}(\overrightarrow{\mathrm{x}}) \cdot \nabla s^{\prime}(\overrightarrow{\mathrm{x}}),
\end{aligned}
$$

where the last term was rewritten from Eq. (3.21) by substituting $1 / 2\left[\nabla s^{\prime}(\overrightarrow{\mathrm{x}} / 2)\right]$ for $\nabla\left[s^{\prime}(\overrightarrow{\mathrm{x}} / 2)\right]$ and then changing variables by $\vec{x} / 2 \rightarrow \vec{x}$.

Now consider the integrations over $s_{\overrightarrow{\mathrm{m}} 0}$. It is convenient to introduce the following definitions:

$$
\begin{aligned}
y_{\overrightarrow{\mathrm{m}}} & =\left(K \rho_{0} / 2\right)^{1 / 2} s_{\overrightarrow{\mathrm{m}} 0} \\
Q(y) & =w^{-1} P\left[\left(K \rho_{0}\right)^{-1 / 2}(2 w)^{1 / 2} y\right] \\
z_{\overrightarrow{\mathrm{m}}} & =\left(K \rho_{0}\right)^{1 / 2}(2 w)^{-1 / 2} 2^{-d / 2} \alpha s^{\prime}\left(\overrightarrow{\mathrm{m}} a_{0} / 2\right) \\
I(z) & =\int_{-\infty}^{\infty} d y \exp \left[-y^{2}-\frac{1}{2} Q(z-y)-\frac{1}{2} Q(z+y)\right] .
\end{aligned}
$$

With these definitions the result of doing the $s_{\overrightarrow{\mathrm{m}} 0}$ integrations in the partition function is to give

$$
\begin{aligned}
Z=\prod_{\overrightarrow{\mathrm{m}}} \prod_{l=1}^{\infty} \int_{-\infty}^{\infty} d s_{\overrightarrow{\mathrm{m}} l} \prod_{\overrightarrow{\mathrm{m}}}\left[\left(K \rho_{0} / 2\right)^{-1 / 2} I\left(z_{\overrightarrow{\mathrm{m}}}\right)\right] \\
\\
\times \exp \left\{-\left(K \alpha^{2} / 8\right) \int_{\overrightarrow{\mathrm{x}}}\left[\nabla s^{\prime}(\overrightarrow{\mathrm{x}})\right]^{2}\right\} .
\end{aligned}
$$

It is convenient to define a new quantity $Z_{1}$ by removing some factors from $Z$, and then define $\mathfrak{H}_{1}$ as the interaction whose integral is $Z_{1}$. In defining $Z_{1}$ it is also convenient to change integration variables from $s_{\overrightarrow{\mathrm{m}} l}$ to $s_{\overrightarrow{\mathrm{m}} l-1}^{\prime}$. Specifically, one writes

$$
Z=Z_{1} \prod_{\overrightarrow{\mathrm{m}}}\left[\left(K \rho_{0} / 2\right)^{-1 / 2} I(0)\right] \prod_{l=1}^{\infty} \prod_{\overrightarrow{\mathrm{m}}} \alpha
$$

and

$$
Z_{1}=\prod_{\overrightarrow{\mathrm{m}}} \prod_{l=0}^{\infty} \int_{-\infty}^{\infty} d s_{\mathrm{m} l}^{\prime} e^{x_{1}} ;
$$

then one has

$$
\mathfrak{H}_{1}=\sum_{\overrightarrow{\mathrm{m}}} \ln \left[I\left(z_{\overrightarrow{\mathrm{m}}}\right) / I(0)\right]-\left(K \alpha^{2} / 8\right) \int_{\overrightarrow{\mathbf{x}}}\left[\nabla s^{\prime}(\overrightarrow{\mathrm{x}})\right]^{2} .
$$

These formulas have been arranged so that the ratio $I\left(z_{\overrightarrow{\mathrm{m}}}\right) / I(0)$ appears in place of just $I\left(z_{\overrightarrow{\mathrm{m}}}\right)$ in the definition of $\mathcal{H}_{1}$. The purpose of this is a practical 
one: In calculations described subsequently, $I\left(z_{\overrightarrow{\mathrm{m}}}\right)$ can turn out to be unmanageably large whereas the ratio is usually not. What one is interested in is not $Z$ itself but the free energy, defined as $V^{-1} \ln Z$ where $V$ is the volume of the system, calculated in the limit $V \rightarrow \infty$. In a volume $V$ there will be $2^{-l d} w V$ position-space boxes associated with momentum shell $l$, or $2^{-l d} w$ boxes per unit volume. Hence one has [from Eq. (3.31)]

$$
\begin{aligned}
F=-\frac{1}{2} w \ln \left(K \rho_{0} / 2\right)+w \ln I(0) \\
+\sum_{l=1}^{\infty} 2^{-l d} w \ln \alpha+V^{-1} \ln Z_{1} .
\end{aligned}
$$

This equation will be discussed further below.

It is convenient to rewrite $\mathfrak{H}_{1}$ by replacing the sum over $\overrightarrow{\mathrm{m}}$ by an integral. This should be a reasonable approximation since the points $\overrightarrow{\mathrm{m}} a_{0} / 2$ for which $s^{\prime}(\overrightarrow{\mathrm{x}})$ is calculated in the sum are closely spaced relative to the distance over which $s^{\prime}(\overrightarrow{\mathrm{x}})$ changes. To be specific we replace $\overrightarrow{\mathrm{m}} a_{0} / 2$ by $\overrightarrow{\mathrm{x}}$; the sum over $\overrightarrow{\mathrm{m}}$ becomes $\left(a_{0} / 2\right)^{-d} \int_{\overrightarrow{\mathrm{x}}}$, where $\left(a_{0} / 2\right)^{d}$ is the volume surrounding the point $\overrightarrow{\mathrm{m}} a_{0} / 2$. Then

$$
\begin{array}{r}
\mathcal{H}_{1}=2^{d} w \int_{\overrightarrow{\mathrm{x}}}\left\{\ln I\left[\left(K \rho_{0} / 2 w\right)^{1 / 2} 2^{-d / 2} \alpha s^{\prime}(\overrightarrow{\mathrm{x}})\right]-\ln I(0)\right\} \\
-\left(K \alpha^{2} / 8\right) \int_{\overrightarrow{\mathrm{x}}}\left[\nabla s^{\prime}(\overrightarrow{\mathrm{x}})\right]^{2}
\end{array}
$$

This means that the form of $\mathcal{H}_{1}$ is the same as the form of $\mathfrak{H}_{0}$. That is, one can write

$$
\mathfrak{H}_{1}=-\int_{\overrightarrow{\mathrm{x}}} P_{1}\left[s^{\prime}(\overrightarrow{\mathrm{x}})\right]-\left(K_{1} / 2\right) \int_{\overrightarrow{\mathrm{x}}}\left[\nabla s^{\prime}(\overrightarrow{\mathrm{x}})\right]^{2},
$$

where

$$
\begin{aligned}
K_{1} & =K \alpha^{2} / 4 \\
P_{1}(s) & =-2^{d} w\left\{\ln I\left[\left(K \rho_{0} / 2 w\right)^{1 / 2} 2^{-d / 2} \alpha s\right]-\ln I(0)\right\} .
\end{aligned}
$$

\section{Recursion Formulas}

One can now set up a recursion formula which allows one to carry out the $s_{\overrightarrow{\mathrm{m}} l}$ integrations for any $l$. To compute the $s_{\overrightarrow{\mathrm{m}} 1}$ integrations, which means the $s_{\overrightarrow{\mathrm{m}} 0}^{\prime}$ integrations, one simply substitutes $P_{1}(s)$ for $P(s)$ and $K_{1}$ for $K$ in the formula for $\mathcal{H}_{0}$ and repeats the calculation which converted $\mathfrak{H}_{0}$ into $\mathcal{H}_{1}$. This will generate a new function $P_{2}(s)$ and a new constant $K_{2}$ which define the interaction $\mathcal{H}_{2}$. The recursion formulas which give $K_{l+1}$ and $P_{l+1}(s)$ in terms of $K_{l}$ and $P_{l}(s)$ will now be obtained. It is convenient in practice to work with the function $Q_{l}(y)$ defined by analogy with Eq. (3.27) to be

$$
Q_{l}(y)=w^{-1} P_{l}\left[\left(2 w / K_{l} \rho_{0}\right)^{1 / 2} y\right] \text {. }
$$

It is also convenient to introduce independent scale factors $\alpha_{l}$ for each $l$, to be fixed later. The recursion formulas are

$$
\begin{aligned}
& K_{l+1}=K_{l} \alpha_{l / 4}^{2}, \\
& I_{l}(z)=\int_{-\infty}^{\infty} d y \exp \left[-y^{2}-\frac{1}{2} Q_{l}(z+y)-\frac{1}{2} Q_{l}(z-y)\right],
\end{aligned}
$$

$Q_{l+1}(y)=-2^{d}\left\{\ln I_{l}\left[2^{-d / 2} \alpha_{l}\left(K_{l} / K_{l+1}\right)^{1 / 2} y\right]-\ln I_{l}(0)\right\}$.

The factor $\left(K_{l} / K_{l+1}\right)^{1 / 2}$ appears in Eq. (3.42) because when Eq. (3.38) is generalized to give $P_{l+1}(s), K$ is replaced by $K_{l}$ inside the function $I_{l}$, while the conversion of $P_{l+1}$ to $Q_{l+1}$ through Eq. (3. 39) involves $K_{l+1}$. Using Eq. (3.40), one can rewrite Eq. $(3.42)$ as

$$
Q_{l+1}(y)=-2^{d}\left[\ln I_{t}\left(2 \times 2^{-d / 2} y\right)-\ln I_{l}(0)\right] .
$$

To complete the recursion formulas, one defines

$$
\begin{aligned}
K_{0} & =K, \\
Q_{0}(y) & =Q(y), \\
\alpha_{0} & =\alpha .
\end{aligned}
$$

There is also a recursion formula for calculating the free energy. There is one point that has to be clarified before the formula can be written down. If the system is confined to a volume $V$, i.e., if $s(\overrightarrow{\mathrm{x}})$ is defined for $\overrightarrow{\mathrm{x}}$ in a volume $V$, then $s^{\prime}(\overrightarrow{\mathrm{x}})$ is defined in a smaller volume, namely, $2^{-d} V$, owing to the scale change going from $s_{1}(\overrightarrow{\mathrm{x}})$ to $s^{\prime}(\overrightarrow{\mathrm{x}})$. Hence when $Z_{1}$ is calculated as integrals over the $s_{\mathrm{m} l}^{\prime}$ one should include only those position-space boxes $\overrightarrow{\mathrm{m}}$ which lie within the volume $2^{-d} V$. It is more convenient, however, to compute $Z_{1}$ over all boxes lying in the original volume $V$; this can be compensated for by multiplying $\ln Z_{1}$ by $2^{-d}$. Define $F_{1}$ to be $F_{1}=V^{-1} \ln Z_{1}$ where $Z_{1}$ is computed as just described; then the term in $F$ due to $Z_{1}$ is $2^{-d} F_{1}$. But the rule for computing $F_{1}$ from $\mathcal{H}_{1}$ is now identical to the rule for computing $F$ from $\mathfrak{F}_{0}$. So we are ready to write a recursion formula involving $F_{l}$ and $F_{l+1}$, namely,

$$
\begin{aligned}
F_{l}=-\frac{1}{2} w \ln \left(K_{l} \rho_{0} / 2\right)+w \ln I_{l}(0) & +2^{-d}\left(1-2^{-d}\right)^{-1} \ln \alpha_{l} \\
& +2^{-d} F_{l+1} .
\end{aligned}
$$

To complete the specification of the recursion formulas, one defines $F=F_{0}$. The recursion formula for $F_{l}$ leads to the following formula for the original free energy $F$ :

$$
\begin{aligned}
F=w \sum_{l=0}^{\infty} 2^{-l d}\left[-\frac{1}{2} \ln \left(K_{l} \rho_{0} / 2\right)+\ln I_{l}(0)\right. \\
\left.+2^{-d}\left(1-2^{-d}\right)^{-1} \ln \alpha_{l}\right] .
\end{aligned}
$$

This formula expresses the free energy as a sum of contributions from each momentum shell. The expression in brackets is the free energy per degree of freedom in shell $l$; the factor $w 2^{-l d}$ in front converts this to a free energy per unit volume. 
So far the constants $\alpha_{l}$ have not been determined. It is not difficult to see that the free energy $F$ is independent of how the $\alpha_{l}$ are chosen: The functions $Q_{t}(y)$ and $I_{l}(z)$ are independent of the $\alpha^{\prime} s$; the constants $K_{l}$ do depend on the $\alpha^{\prime}$ s, but $Q_{l}(y)$ and $I_{l}(z)$ do not depend on the $K$ 's. In the free energy itself any change in the $\alpha^{\prime} s$ is compensated by corresponding changes in the $K^{\prime}$ s. The $\alpha$ 's will therefore be left arbitrary for now.

\section{Interpretation of Recursion Formulas}

The recursion formulas for carrying out the integration over the $l$ th momentum shell have now been written out. The next step is to clarify the significance of these formulas, and to relate them to the general ideas of the renormalization group.

Define $s_{t}(\overrightarrow{\mathrm{x}})$ to be

$$
s_{l}(\overrightarrow{\mathrm{x}})=\sum_{\overrightarrow{\mathrm{m}}} \sum_{l_{1}=l}^{\infty} \psi_{\overrightarrow{\mathrm{m}} l_{1}}(\overrightarrow{\mathrm{x}}) s_{\overrightarrow{\mathrm{m}} l_{1}}
$$

where the $s_{\overrightarrow{\mathrm{m}} l}$ are the original phase-space cell variables. The field $s_{l}(\overrightarrow{\mathrm{x}})$ is the contribution to the original field $s(\overrightarrow{\mathrm{x}})$ from degrees of freedom with momenta $\leq 2 \times 2^{-l}$. The field $s_{l}(\overrightarrow{\mathrm{x}})$ is constant over distances small compared to $2^{l}$ (in units of the original lattice spacing); for qualitative purpose the field $s_{l}(\overrightarrow{\mathrm{x}})$ can be represented by its values on a lattice with lattice spacing $\sim 2^{l}$. The effective interaction $\mathcal{H}_{l}$ resulting from integrating the variables $s_{\overrightarrow{\mathrm{m}} 0}, s_{\overrightarrow{\mathrm{m}} 1}, \ldots, s_{\overrightarrow{\mathrm{m}} l-1}$ could have been written as a functional of $s_{l}(\overrightarrow{\mathrm{x}})$. However, it was decided to express $\mathcal{H}_{l}$ as a functional of a scaled field variable, which we shall denote $s_{c l}(\overrightarrow{\mathrm{x}})$. The relation of $s_{l}(\overrightarrow{\mathrm{x}})$ to $s_{c l}(\overrightarrow{\mathrm{x}})$ is

$$
s_{l}(\overrightarrow{\mathrm{x}})=2^{-l d / 2} \alpha_{0} \alpha_{1} \alpha_{2} \cdots \alpha_{l-1} s_{c l}\left(2^{-l} \overrightarrow{\mathrm{x}}\right) .
$$

The product $\alpha_{0} \cdots \alpha_{l-1}$ arises because there are $l$ changes of scale involved in the definition of $s_{c l}\left(2^{-l} \overrightarrow{\mathrm{x}}\right)$. The scaled field $s_{c l}(\overrightarrow{\mathrm{x}})$ is representable, qualitatively speaking, by its values on a lattice with unit lattice spacing. In terms of $s_{c l}(\overrightarrow{\mathrm{x}})$, the effective interaction is

$$
\mathfrak{H}_{l}=-\int_{\overrightarrow{\mathrm{x}}} P_{l}\left[s_{c l}(\overrightarrow{\mathrm{x}})\right]-K_{l} / 2 \int_{\overrightarrow{\mathrm{x}}}\left[\nabla s_{c l}(\overrightarrow{\mathrm{x}})\right]^{2},
$$

where

$$
P_{l}(s)=w Q_{l}\left[\left(K_{l} \rho_{0} / 2 w\right)^{1 / 2} s\right] .
$$

The effective interaction $\mathfrak{H}_{l}$ is analogous to the effective Ising model discussed in I for block size $L=2^{l}$. The interaction $\mathfrak{K}_{l}$ describes the interactions of low momentum degrees of freedom, to be precise degrees of freedom with wavelengths of order $2^{l}$ or larger. The field variable $s_{l}(\overrightarrow{\mathrm{x}})$ is analogous to block spin variables of $I$ for block spacing $L=2^{l}$. By introducing the scaled variable $s_{c l}(\vec{x})$ one has eliminated all reference to the new lattice spacing in $\mathscr{H}_{l}$; the only way $\mathfrak{H}_{l}$ is distinguishable from the original interaction $\mathcal{F}_{0}$ is if $K_{l}$ is different from $K$ or if $P_{l}(s)$ is a different function from $P(s)$. This is analogous to the result of I that the block Hamiltonian could be distinguished from the original Hamiltonian only if the coupling parameters $K_{L}$ and $h_{L}$ of the block Hamiltonian were different from the original constants $K$ and $h$. Having an $l$-dependent function $P_{l}(s)$ is equivalent to having an infinite number of $l$-dependent constants. For example, one might try to represent $P_{l}(s)$ by a power series expansion $\sum_{n=0}^{\infty} f_{n l} s^{2 n}$. The constants $f_{n l}(0 \leq n \leq \infty)$ are an infinite set of $l$-dependent constants.

The recursion formulas determine $P_{l+1}(s)$ and $K_{l+1}$ given $K_{l}, \alpha_{l}$, and the function $P_{l}(s)$. (The scale factor $\alpha_{l}$ is analogous to the scale factor introduced in I when a block spin was rescaled to have the values \pm 1 instead of $\pm L^{3}$; see below.) The recursion formulas are analogous to the renormalization-group differential equations of $\mathrm{I}$. More precisely the recursion formulas are analogous to the finite transformation giving $K_{2 L}$ and $h_{2 L}$ in terms of $K_{L}$ and $h_{L}$, since the effective lattice spacing is doubled when $l \rightarrow l+1$.

The basic idea of I was that at the critical point the coupling parameters of the effective Ising model should approach a fixed point $\left(K_{L} \rightarrow K_{c}, h_{L}=0\right)$ when $L \rightarrow \infty$. This continued to be true when there was an "irrelevant" parameter $q_{L}$ in the effective Ising interaction. In this paper the renormalization group involves an infinite number of irrelevant parameters. One can still hypothesize that the critical point corresponds to a fixed point, although, as was made clear at the end of $I$, this is not guaranteed.

A fixed point would be a solution of the recursion formulas independent of $l$, e.g., $P_{l}(s)=P_{c}(s)$ and $K_{l}=K_{c}$ for all $l$. It is easy enough to make $K_{l}$ be a constant: We simply define $\alpha_{l}=2$ for all $l$. So the crucial problem is whether there exists a function $P_{c}(s)$ which solves the recursion formula. In practice this means there should be a function $Q_{l}(y) \equiv Q_{c}(y)$ solving Eqs. (3.41) and (3.43).

In I the effective coupling parameter $K_{L}$ was independent of $L$ only at the critical point, whereas now one can fix $K_{l}=K$ independently of $l$ simply by choosing $\alpha_{l}=2$ for all $l$. The reason for this discrepancy is that in I the scale factors analogous to $\alpha_{l}$ were chosen to fix the order of magnitude of the scaled block spins (the scaled spins had values \pm 1 ). In principle one could choose the constants $\alpha_{l}$ to fix the order of magnitude of the scaled spin functions $s_{c l}(\overrightarrow{\mathrm{x}})$ but in practice it is awkward to do so. So in this paper one keeps $K_{l}$ fixed but the order of magnitude of $s_{c l}(\overrightarrow{\mathrm{x}})$ is allowed to float. At a fixed point the order of magnitude of $s_{c l}(\vec{x})$ will be independent of $l$ because it can only change with $l$ if $Q_{l}(y)$ changes with $l$ (see below).

To continue this analysis of the significance of 
the recursion formulas, it is necessary to have a qualitative formula for the spin-spin correlation function $g(\vec{k})$. The calculation that follows will give only a rough order of magnitude for $g(\vec{k})$ but will be sufficient for the purposes of this paper. A preliminary discussion of $g(\overrightarrow{\mathbf{k}})$ was given in Sec. II C; Eqs. (2.16) and the continuum form of (2.15) are still valid. It will still be true that the products $s_{\overrightarrow{\mathrm{m}} l_{1}} s_{0 l}$ average to zero unless $\overrightarrow{\mathrm{m}}=0$ and $l_{1}=l$, but this must be proven again. Consider the expectation value of $s_{\overrightarrow{\mathrm{m}} l} s_{\overrightarrow{\mathrm{m}}_{1} l_{1}}$ for arbitrary $\overrightarrow{\mathrm{m}}, l, \overrightarrow{\mathrm{m}}_{1}$, and $l_{1}$. Let $l_{1}$ be greater than or equal to $l$. The thermodynamic expectation value is

$$
\left\langle s_{\overrightarrow{\mathrm{m}} l} s_{\overrightarrow{\mathrm{m}}_{1} l_{1}}\right\rangle=Z^{-1} \prod_{\overrightarrow{\mathrm{m}} 2} \prod_{l 2=0}^{\infty} \int_{-\infty}^{\infty} d s_{\overrightarrow{\mathrm{m}}_{2} l_{2}}\left(s_{\overrightarrow{\mathrm{m}} l} s_{\overrightarrow{\mathrm{m}}_{1} l_{1}} e^{\mathfrak{H}_{0}}\right) .
$$

The integrals for $l_{2}<l$ can be carried out in the same fashion as in the calculation of $Z$. When one comes to do the integrations for $l_{2}=l$ one gets one integral with an extra factor $s_{\overrightarrow{\mathrm{m}} l}$ in it. (Assume for now that $l_{1}>l$ so $s_{\overrightarrow{\mathrm{m}}_{1} l_{1}}$ is not involved in the integrations for $l_{2}=l$.) The actual integration variable $y$ is a scaled form of $s_{\overrightarrow{\mathrm{m}} l}$; if one traces through the changes in scale one finds $s_{\overrightarrow{\mathrm{m}} l}=\alpha_{0} \alpha_{1} \cdots \alpha_{l-1}$ $\times\left(K_{l} \rho_{0} / 2\right)^{-1 / 2} y$. Now the integral over $s_{\overrightarrow{\mathrm{m}} l}$ in $Z$ reduces to the form given in Eq. (3.41) where $z$ is a variable depending on degrees of freedom with $l_{2}>l$. With the extra factor $s_{\overrightarrow{\mathrm{m}} l}$ included this integral becomes

$$
\begin{aligned}
& \alpha_{0} \alpha_{1} \cdots \alpha_{l-1}\left(K_{l} \rho_{0} / 2\right)^{-1 / 2} \int_{-\infty}^{\infty} d y y \\
& \quad \times \exp \left[-y^{2}-\frac{1}{2} Q_{l}(z-y)-\frac{1}{2} Q_{l}(z+y)\right] .
\end{aligned}
$$

This integral vanishes for any $z$ because the integrand is odd in $y$. This means that the expectation value of $s_{\overrightarrow{\mathrm{m}} l} s_{\overrightarrow{\mathrm{m}}_{1} l_{1}}$ vanishes unless $\overrightarrow{\mathrm{m}}_{1}=\overrightarrow{\mathrm{m}}$ and $l_{1}=l$. Thus one should consider the expectation value $\left\langle s_{\overrightarrow{\mathrm{m}} l}^{2}\right\rangle$. In this case the integral over $s_{\overrightarrow{\mathrm{m}} l}$ becomes

$$
\begin{aligned}
\alpha_{0}^{2} \alpha_{1}^{2} \cdots \alpha_{l-1}^{2} & \left(K_{l} \rho_{0} / 2\right)^{-1} \int_{-\infty}^{\infty} d y y^{2} \\
& \times \exp \left[-y^{2}-\frac{1}{2} Q_{l}(z-y)-\frac{1}{2} Q_{l}(z+y)\right] .
\end{aligned}
$$

It is convenient to define

$$
\begin{aligned}
R_{l}(z)= & {\left[I_{l}(z)\right]^{-1} \int_{-\infty}^{\infty} d y y^{2} } \\
& \times \exp \left[-y^{2}-\frac{1}{2} Q_{l}(z-y)-\frac{1}{2} Q_{l}(z+y)\right] .
\end{aligned}
$$

The function $R_{l}(z)$ is the expectation value of $y^{2}$ for fixed $z$. If $R_{l}(z)$ were to be independent of $z$, then the integral containing $s_{\overrightarrow{\mathrm{m}} l}^{2}$ would only differ by a constant factor from the integral without $s_{\overrightarrow{\mathrm{m}} l}^{2}$; in this case the average $\left\langle s_{\overrightarrow{\mathrm{m}} l}^{2}\right\rangle$ would be simply $\alpha_{0}^{2} \cdots \alpha_{l-1}^{2}\left(K_{l} \rho_{0} / 2\right)^{-1} R_{l}$. When $R_{l}(z)$ does depend on $z$, then to compute $\left\langle s_{\overrightarrow{\mathrm{m}} \imath}^{2}\right\rangle$ one must not only com- pute the integral over $y$ that gives $R_{l}(z)$; one must also compute integrals over those degrees of freedom for $l_{2}>l$ which are contained in $z$. That is, there will be an integral for $l_{2}=l+1$ which differs from Eq. $(3.41)^{16}$ by including the function $R_{l}$ \{to be precise, $\left.R_{l}\left[2 \times 2^{-d / 2}(z+y)\right]\right\}^{17}$; the presence of $R_{l}$ will change the dependence on $z$ of this integral, so in turn an integral for $l_{2}=l+2$ will also differ from Eq. (3.41), and so forth. The reason for this complication is the coupling between the $l$ th momentum shell and shells for $l_{2}>l$. The expectation value $\left\langle s_{\overrightarrow{\mathrm{m}} l}^{2}\right\rangle$ cannot be determined until this coupling is taken into account and the only way this can be done is by integrating the shells with larger $l_{2}$.

For an order-of-magnitude calculation, we shall substitute $R_{i}(0)$ for $R_{l}(z)$ and not carry out subsequent integrations involving $R_{l}$. This substitution would be invalid if $R_{l}(z)$ were to change by orders of magnitude from $R_{l}(0)$ over the range of $z$ that is important in subsequent integrations. In the calculations of this paper $R_{l}(z)$ does not change drastically over the important range of $z$. However, it is a nontrivial assumption that $R_{l}(z)$ does not change drastically and one must check this assumption every time it is used.

Now one can write down the order-of-magnitude formula for $g(\overrightarrow{\mathrm{k}})$. If $\overrightarrow{\mathrm{k}}$ lies in momentum shell $l$ then [from Eqs. (2.16), (3.5), and (3.8)] $g(\overrightarrow{\mathrm{k}})$ $=\left\langle s_{0 l}^{2}\right\rangle$. Replacing $R_{l}(z)$ by $R_{l}(0)$, one gets

$$
g(\overrightarrow{\mathrm{k}}) \simeq \alpha_{0}^{2} \cdots \alpha_{l-1}^{2}\left(K_{l} \rho_{0} / 2\right)^{-1} R_{l}(0) .
$$

With all $\alpha$ 's equal to 2 and $K_{l}=K$ this gives

$$
g(\overrightarrow{\mathrm{k}}) \sim 2^{2 t} R_{l}(0)
$$

(dropping all constants which do not depend on $l$ ). A consequence of this formula is that if $Q_{l}(y)-Q_{c}(y)$ at the critical point then $g(\vec{k}) \sim 2^{2 l}$ when $|\vec{k}| \sim 2^{-t}$, i. e., $g(\vec{k}) \sim k^{-2}$. This is in contradiction with the previous analysis neglecting coupling terms, which gave a different critical exponent for dimension $d<4$. In the analysis for $d<4$ neglecting all coupling terms, it was found that the term proportional to $s_{\overrightarrow{\mathrm{m}} l}^{2}$ was negligible compared to the $s_{\overrightarrow{\mathrm{m}} l}^{4}$ term. This was true provided $l$ was large and one was at the critical temperature. In the present analysis the $s_{\mathrm{m} l}^{2}$ term has become the explicit $y^{2}$ term in the integral for $I_{l}(z)$ while the $s_{\mathrm{m} l}^{4}$ term has been replaced by the $Q_{l}(y \pm z)$ terms. The $y^{2}$ term would be negligible too if the function $Q_{l}(y)$ were to increase rapidly with $y$ so as to make $R_{l}(0) \ll 1$. This does not happen in practice, which is fortunate. If one could neglect the $y^{2}$ term at the critical point, there would be no need for its presence. But the absence of the $y^{2}$ term, for all $l$, would mean the $\nabla s(x) \cdot \nabla s(x)$ term is absent in the original interaction. Then one could set $K=0 ; K$ also appears in $P(s)$ but there any change in $K$ can be 
compensated for by changing $r$. So if the $y^{2}$ term is negligible at the critical point, one could get critical behavior even in the absence of spin-spin coupling, simply by choosing $r$ suitably. This idea is absurd. So for the present analysis to make sense the $y^{2}$ term cannot be negligible at the critical point. This discussion shows that the previous analysis neglecting all coupling terms cannot be correct, since according to the previous analysis one could get critical behavior neglecting the spin-spin interaction terms.

According to the present analysis $g(\vec{k})$ behaves as $k^{-2}$ at the critical point for any dimension $d$, whereas other calculations give $g(\vec{k}) \sim k^{42+n}$ with $\eta=0.25$ for two dimensions and $\eta \simeq 0.06$ in three dimensions. ${ }^{18}$ However, the present analysis is only qualitative. It will be shown later that some of the terms neglected in the present analysis can lead to a nonzero $\eta .^{10}$ Since the neglected terms are supposed to be small, they should lead to a small $\eta$. This is consistent with the expected numbers 0.25 and 0.06 .

\section{SOLUTIONS OF RECURSION FORMULAS}

\section{A. Expectations}

It will be useful to have some expectations about the solution of the recursion formulas for $Q_{t}(z)$. These expectations will be checked later against numerical solutions of the recursion formulas. If one is above the critical temperature then $g(\vec{k})$ should be finite for $\overrightarrow{\mathrm{k}}-0$. This requires that $R_{t}(0)$ (see Sec. III D) behaves as $2^{-2 l}$ for large enough $l$. If one goes back to the analysis neglecting all coupling terms, one predicts that (except at the critical point) the interaction becomes Gaussian for large $l$. If this is so then $Q_{l}(z)$ must be proportional to $2^{2 l} z^{2}$ for large $l$ in order that $R_{l}(0)$ behave as $2^{-2 l}$. At the critical point one hopes that $Q_{l}(z)$ approaches a limit $Q_{c}(z)$. Temperatures below the critical temperature will not be discussed in detail. Near the critical temperature one can use a linearized form of the recursion formulas similar to the linearized renormalization-group equations discussed in I. If $Q_{t}(z)-Q_{c}(z)$ is small then one expects $Q_{t+1}(z)-Q_{c}(z)$ to be a linear functional of $\left[Q_{t}(z)-Q_{c}(z)\right]$, namely,

$$
Q_{t+1}(z)-Q_{c}(z) \simeq \int_{-\infty}^{\infty} T(z, y)\left[Q_{t}(y)-Q_{c}(y)\right] .
$$

From Eqs. (3.41) and (3.43) one can get an explicit formula for $T$ in terms of the function $Q_{c}$. Let $x$ be $2 \times 2^{-d / 2} z$; then

$$
\begin{aligned}
T(z, y)= & 2^{d}\left(\frac{\exp \left[-(y-x)^{2}-\frac{1}{2} Q_{c}(y)-\frac{1}{2} Q_{c}(2 x-y)\right]}{I_{c}(x)}\right. \\
& \left.-\frac{\exp \left[-y^{2}-\frac{1}{2} Q_{c}(y)-\frac{1}{2} Q_{c}(-y)\right]}{I_{c}(0)}\right)
\end{aligned}
$$

where $I_{c}(z)$ is $I_{t}(z)$ calculated for $Q_{l}(y) \equiv Q_{c}(y)$. As discussed in $I$, the linearized recursion formula should have one exponentially growing solution reflecting the instability of the fixed point to changes in temperature, and a second exponentially growing solution reflecting the instability to adding a magnetic field. An exponentially growing solution corresponding to the temperature instability should have the form

$$
Q_{l}(z)-Q_{c}(z)=2^{l y K} q(z),
$$

where $y_{K}$ is a constant. [The $l$ dependence is written $2^{l y_{K}}$ because $2^{l}$ is equivalent to the variable $L$ of $I$ so $2^{t_{K}}$ is equivalent to $L^{y_{K}}$ and Eq. (4.3) is analogous to Eq. (6) of 1 . Here the symbol $y_{K}$ is used for the exponent to distinguish it from the integration variable $y$.] The function $q(z)$ must be an eigenfunction of $T$ :

$$
2^{y K} q(z)=\int_{-\infty}^{\infty} T(z, y) q(y) d y \text {. }
$$

From symmetry arguments one would expect the temperature instability to be associated with an eigenfunction $q(z)$ even in $z$, while the magnetic field instability would have an odd eigenfunction.

The magnetic unstable solution can be given explicitly; it is

$$
Q_{l}(z)-Q_{c}(z)=\left(2^{l \times K}\right) z
$$

where

$$
x_{K}=d / 2+1 \text {. }
$$

If one solves the nonlinear recursion formulas for $Q_{l}(z)$ for temperatures slightly above critical and no magnetic field, then one expects that

$$
Q_{1}(z) \simeq\left(K_{c}-K\right) 2^{l y K} q(z)+Q_{c}(z),
$$

where $K_{c}$ is the critical value of $K$ and there are the following restrictions: (i) $q(z)$ is suitably normalized to eliminate a constant of proportionality; (ii) $l$ must be large enough to eliminate initial transient terms corresponding to exponentially decreasing solutions of the linearized recursion formula (see I); (iii) $l$ must not be so large that $Q_{l}(z)$ $-Q_{c}(z)$ is large. For $K$ near $K_{c}$ and very large $l$, $Q_{l}(z)$ is no longer near $Q_{c}(z)$, so $Q_{l}(z)-Q_{c}(z)$ is no longer a solution of the linearized recursion formula. For very large $l$ one expects

$$
Q_{l}(z) \approx f\left(K_{c}-K\right) 2^{2 t} z^{2}
$$

where $f$ is a function to be determined. Now for intermediate values of $l$, for which Eq. (4.7) holds, one has a translational symmetry, namely,

$$
Q_{l+1}\left(z, K_{c}-K\right) \simeq Q_{l}\left(z, 2^{y_{K}}\left(K_{c}-K\right)\right) .
$$

This kind of symmetry is preserved by the nonlinear recursion formula so it should hold also for very large $l$; from Eq. (4.8) one gets 


$$
4 f\left(K_{c}-K\right)=f\left(2^{y} K\left(K_{c}-K\right)\right) \text {. }
$$

The solution of this equation is

$$
f(\epsilon)=\epsilon^{2 / y_{K}} f_{1}(\epsilon) \text {, }
$$

with $f_{1}(\epsilon)$ periodic in $\ln \epsilon$ with period $y_{K} \ln 2$; in other words $f_{1}(\epsilon)$ is determined for small $\epsilon$ if it is known for $\epsilon \sim 1$, which means that for order-ofmagnitude purposes

$$
f(\epsilon) \sim \epsilon^{2 / y_{K}} .
$$

One can now get the dependence of the magnetic susceptibility $\chi$ on $\epsilon=K_{c}-K$. The susceptibility is the correlation function $g(\vec{k})$ evaluated for $k=0$. This means, qualitatively, $\chi$ is $2^{2 l} R_{l}(0)$ in the limit $l \rightarrow \infty$. In this limit, using the form (4.8) for $Q_{i}(z)$, one gets

$$
\chi \sim 1 / f(\epsilon) \text {. }
$$

Therefore $\chi$ behaves as $\epsilon^{-2 / y_{K}}$. One generally writes the behavior of $\chi$ as $\epsilon^{-\gamma}$, so this analysis gives $\gamma$ as $2 / y_{K}$.

The correlation length $\xi$ can be defined qualitatively as the inverse of the value of $k$ below which $g(\overrightarrow{\mathrm{k}})$ is of order $g(0)$. This means that $\xi \sim 2^{l_{M}}$ where $l_{M}$ is the lowest value of $l$ for which the large- $l$ approximation (4.8) holds. If there is not a large gap between the breakdown of the linear approximation (4.7) and the onset of the large- $l$ approximation (4.8), then $l_{M}$ is given qualitatively by the value of $l$ for which $Q_{l}(z)-Q_{c}(z)$ is of order 1 , namely,

$$
2^{l_{M} y_{K}} \in \sim 1
$$

or

$$
\xi \sim 2^{l_{M} \sim \epsilon^{1 / y_{K}}} .
$$

The correlation length is usually written $\xi \sim \epsilon^{-\nu}$ so one expects $\nu=1 / y_{K}$.

In summary, one has three exponents which can now be determined, namely, $\eta$ (already found to be zero), $\gamma$, and $\nu$. There are others one would like to obtain but they will not be discussed here, because this paper is already too long.

\section{B. Gaussian Model}

Now solutions of the recursion formula for $Q_{l}(y)$ will be discussed. First consider the Gaussian model. In this case $Q(y)$ has the form

$$
Q(y)=r_{0} y^{2},
$$

where, from Eqs. (3.12) and (3.27),

$$
r_{0}=\left(2 / K \rho_{0}\right)(r-K d)
$$

and $K$ and $r$ are the coefficients in the original lattice model [Eq. (1.2)]. Given that $Q(y)$ is quadratic in $y$, it is easily seen that $Q_{l}(y)$ is also quadratic:

$$
Q_{l}(y)=r_{l} y^{2} \text {. }
$$

This gives

$$
I_{l}(z)=\pi^{1 / 2}\left(1+r_{l}\right)^{-1 / 2} e^{-r} t^{z^{2}} .
$$

Equation (3.43) now gives

$$
r_{l+1}=4 r_{l}
$$

So one has

$$
r_{l}=4^{l} r_{0}
$$

To compute the correlation function one must compute $R_{l}(0)$; from Eq. (3.54) this is

$$
R_{l}(0)=\frac{1}{2} \frac{1}{1+r_{l}}
$$

From Eq. (3.56) the correlation function is

$$
g(\overrightarrow{\mathrm{k}}) \sim \frac{1}{k^{2}} \frac{1}{1+r_{0} / k^{2}}=\frac{1}{r_{0}+k^{2}}
$$

(for given $k=|\overrightarrow{\mathbf{k}}|, l$ is determined by the requirement $\left.2^{-1} \sim k\right)$. This is the expected form of the correlation function for the Gaussian model. The critical point occurs at $r_{0}=0$. For $r_{0}=0, Q_{\imath}(y)$ approaches a fixed function $Q_{c}(y)$ for $l \rightarrow \infty$, in fact $Q_{l}(y) \equiv Q_{c}(y)$ for all $l$. The function $Q_{c}(y) \equiv 0$ for all $y$. This function defines a fixed point of the renormalization group which will be called the Gaussian fixed point. [The requirement $r_{0}=0$ determines the critical value of the spin-spin coupling constant $K$ to be $r / d$, from Eq. (4.17).]

The exponentially growing solution near the fixed point corresponding to the temperature instability is given by Eqs. (4.18) and (4.21), which in turn give $y_{K}=2$. Therefore $\gamma=1$ and $\nu=0.5$; these are the exact exponents for the Gaussian model.

\section{Perturbations of Gaussian Model}

The next step is to look at small departures from the Gaussian model. Consider first the addition of a small quartic term. Then $Q(y)$ has the form

$$
Q(y)=r_{0} y^{2}+\lambda_{0} y^{4}
$$

where $\lambda_{0} \ll r_{0}$. [In terms of the original quartic constant $\lambda, \lambda_{0}$ is $\left(2 / K \rho_{0}\right)^{2} w \lambda$.] Calculation shows that to second order in $\lambda_{0}, Q_{1}(y)$ is also quartic:

$$
Q_{1}(y)=r_{1} y^{2}+\lambda_{1} y^{4},
$$

where $r_{1}$ and $\lambda_{1}$ depend on $r_{0}$ and $\lambda_{0}$. More generally, one has

$$
Q_{l}(y)=r_{l} y^{2}+\lambda_{l} y^{4}
$$

provided one calculates the recursion formula for $Q_{l}$ only to second order in $\lambda_{l-1}$. Given a $Q_{l}(y)$ of this form one has, to order $\lambda_{l}^{2}$,

$$
\begin{aligned}
I_{l}(z) \simeq & \int_{-\infty}^{\infty} d y\left[1-\lambda_{l}\left(y^{4}+6 z^{2} y^{2}\right)+\frac{1}{2} \lambda_{l}^{2}\left(y^{4}+6 z^{2} y^{2}\right)^{2}\right] \\
& \times \exp \left(-y^{2}-r_{l} y^{2}-r_{l} z^{2}-\lambda_{l} z^{4}\right)
\end{aligned}
$$

Let $q_{l}=\left(1+r_{l}\right)^{-1}$. Then one has 


$$
\begin{aligned}
I_{l}(z) \simeq\left(q_{l} \pi\right)^{1 / 2}\left[1-\lambda_{l}\left(\frac{3}{4} q_{l}^{2}+\frac{6}{2} q_{l} z^{2}\right)\right. \\
\left.+\lambda_{l}^{2}\left(\frac{105}{32} q_{l}^{4}+\frac{12 \times 15}{16} q_{l}^{3} z^{2}+\frac{36 \times 3}{8} q_{l}^{2} z^{4}\right)\right] \\
\quad \times \exp \left(-r_{l} z^{2}-\lambda_{l} z^{4}\right) . \quad(4.28)
\end{aligned}
$$

From Eqs. (4.28 and (3.43) one obtains a quartic form for $Q_{l+1}(y)$, neglecting terms of order $\lambda_{l}^{3}$, with

$$
\begin{aligned}
& r_{l+1}=4\left(r_{l}+3 q_{l} \lambda_{l}-9 q_{l}^{3} \lambda_{l}^{2}\right), \\
& \lambda_{l+1}=16 \times 2^{-d}\left(\lambda_{l}-9 q_{l}^{2} \lambda_{l}^{2}\right) .
\end{aligned}
$$

Consider first the recursion formula for $\lambda_{l+1}$. For dimension 3 or less, $\lambda_{l+1}$ is larger than $\lambda_{l}$ (if $\lambda_{l}$ is small enough). This means the Gaussian fixed point is unstable to non-Gaussian perturbations; if one starts with a small term $\lambda_{0} y^{4}$, the corresponding term $\lambda_{l} y^{4}$ increases with $l$ until a perturbation expansion in $\lambda_{l}$ no longer makes sense. In this case the Gaussian fixed point is analogous to the doubly unstable point discussed in I in the presence of an irrelevant variable. In this case the Gaussian fixed point is relevant to the critical behavior of the model (1.2) only if $\lambda=0$. On the other hand, for 5 dimensions or higher, $\lambda_{l}$ decreases with $l$ so that if one starts with small but finite $\lambda$, the quartic term disappears as $l$ increases: One approaches the Gaussian fixed point as $l \rightarrow \infty$. To be precise, to approach the Gaussian fixed point requires that $\lambda_{l}$ and $r_{l}$ both go to 0 as $l \rightarrow \infty$. This is automatic for $\lambda_{l}$, if $d \geq 5$. For $r_{l} \rightarrow 0$ also one must choose $r_{0}$ correctly. From the requirement that $r_{l} \rightarrow 0$ for $l \rightarrow \infty$ one can compute $r_{l}$ for finite $l$ from the recursion formula (4.29) rewritten to give $r_{l}$ in terms of $r_{l+1}$. In particular this allows one to compute $r_{0}$ and therefore to determine the critical value of $K$. If one computes only to first order in $\lambda_{l}$, one obtains

$$
r_{l}=-3 \sum_{n=l}^{\infty} 4^{-(n-l)} q_{n} \lambda_{n}
$$

If $\lambda_{l}$ is small for all $l$ then so is $r_{l}$, so $q_{l} \simeq 1$. Then

$$
r_{l} \simeq-3 \lambda_{l}\left(1-4 \times 2^{-d}\right)^{-1} \text {. }
$$

This equation, for $l=0$, can be rewritten

$$
r-K d=-3\left(1-4 \times 2^{-d}\right)^{-1}\left(2 / K \rho_{0}\right) w \lambda \text {. }
$$

This equation determines the critical value of $K$, to first order in $\lambda$.

In four dimensions the recursion formula for $\lambda_{1}$ is

$$
\lambda_{l+1} \simeq \lambda_{l}-9 q_{l}^{2} \lambda_{l}^{2} .
$$

In this case $\lambda_{l}$ decreases with $l$, but rather slowly. One still finds that $r_{l}$ is small at the critical point, in fact Eqs. (4.32) still holds, so one has

$$
\lambda_{l+1} \simeq \lambda_{l}-9 \lambda_{l}^{2} .
$$

For small $\lambda_{l}, \lambda_{l}$ changes slowly with $l$ and it is a good approximation to rewrite this recursion formula as a differential equation:

$$
\frac{d \lambda_{l}}{d l} \simeq-9 \lambda_{l}^{2}
$$

The solution of this equation is

$$
\lambda_{l}=\lambda_{0}\left(1+9 l \lambda_{0}\right)^{-1}
$$

so $\lambda_{l}$ goes to zero as $l^{-1}$.

Thus one confirms the analysis neglecting all coupling terms in that the critical behavior of the model with a small quartic term is the same as the critical behavior of the Gaussian model in 5 dimensions and higher. ${ }^{20}$ In four dimensions the critical behavior is close to that of the Gaussian model, but the persistence of the quartic term leads to some departures which will not be discussed here. In three dimensions or less, one has to look for a non-Gaussian fixed point to describe the critical behavior of non-Gaussian models.

\section{Numerical Solution for Three Dimensions}

To find the critical behavior in three dimensions for the non-Gaussian model, the recursion formula for $Q_{l}(z)$ was calculated numerically. The function $Q_{l}(z)$ was calculated on a uniformly spaced mesh with 41 points from $z=0$ to $z=4$. Linear interpolation was used to compute $Q_{l}(z)$ for points in between the mesh points. For $z>4, Q_{l}(z)$ was approximated by the form

$$
Q_{l}(z) \simeq Q_{l}(4)(z / 4)^{6} .
$$

The exact form used for $z>4$ did not seem to affect the calculation much. The integral over $y$ was calculated by neglecting the region $y>3.2$ and dividing the region $-3.2<y<3.2$ into 30 equally spaced intervals. In each interval the integral was calculated using the two-point Gaussian integration formula. ${ }^{21}$ All calculations were performed in doubleprecision arithmetic on the Wilson Synchrotron Laboratory PDP-10 computer.

The initial function $Q(z)$ was chosen to have the form of Eq. (4.24); $\lambda_{0}$ was fixed at 0.5 and $r_{0}$ was varied to locate the critical point. It was not difficult to locate the critical value of $r_{c}$ for $r_{0}$. For $r_{0}>r_{c}$ the values of $Q_{l}(z)$, for fixed $z$, went rapidly to $+\infty$ as $l \rightarrow \infty$; for $r_{0}<r_{c}$ the values of $Q_{l}(z)$ were erratic. In practice, $r_{c}$ was found by finding the sequence of values $r_{c t}$ for which the $l$ th function $Q_{l}(z)$ satisfies $Q_{l}(1.5)=0$. This sequence appeared to be converging rapidly with increasing $l$ : The differences $r_{c l}-r_{c}$ as computed numerically are shown in Table II. The value of $r_{c}$ was found to be 
TABLE II. Values of $r_{c l}-r_{c}$ vs $l$. The quantity $r_{c l}$ is defined as the value of $r$ for which $Q_{l}(1.5)=0$.

\begin{tabular}{ll}
\hline \hline$l$ & $r_{c l}-r_{l}$ \\
\hline 2 & 0.024 \\
3 & 0.015 \\
4 & 0.0046 \\
5 & 0.0015 \\
6 & 0.00047 \\
7 & 0.00015 \\
8 & 0.000048 \\
\hline \hline
\end{tabular}

$$
r_{c} \simeq-1.66678
$$

When $r_{0} \simeq r_{c}$ it was found that the function $Q_{l}(z)$, as $l$ increased, first approached a critical function $Q_{c}(z)$, then for very large $l$ moved away from $Q_{c}(z)$, just as predicted earlier. The critical function $Q_{c}(z)$ is plotted in Fig. 4.

The quantities $Q_{l}(1.5)$ are plotted versus $l$ in Fig. 5 for two values of $r_{0}$. Comparison of these solutions with Eq. (4.7) gives excellent agreement for intermediate values of $l$, with

$$
y_{K}=1.65 \text {. }
$$

To show this, the quantity $2^{-l y_{K}}\left[Q^{l}(1.5)-Q_{c}(1.5)\right] /$ $\left(r_{c}-r_{0}\right)$ is tabulated in Table III versus $l$. It is also found that $Q_{l}(z)$ behaves like Eq. (4.8) for very large $l$, and to illustrate this the quantity $2^{-2 l}\left(r_{c}-r_{0}\right)^{-2 / y} K Q_{l}(1.5)$ is also tabulated in Table III. One can see from Table III that one or the other of Eqs. (4.7) and (4.8) holds beyond the initial transient range of $l$, except for one or two values of $l$. So the previous argument giving the exponent $\nu$ as $1 / y_{K}$ is valid, as is the result $\gamma=2 / y_{K}$. From Eq. (4.40) one gets

$$
\begin{aligned}
& \nu=0.609, \\
& \gamma=1.218 .
\end{aligned}
$$

The predictions of numerical calculations of the three dimensional Ising model are ${ }^{22} \nu=0.643$, $\gamma=1.25$; there is impressive agreement between the Ising-model results and the calculations of this paper.

One final note. According to expectations [see Eqs. (4.8) and (4.11)], the function

$$
f_{1}\left(r-r_{0}\right)=\lim _{l \rightarrow \infty} 2^{-2 l}\left(r_{c}-r_{0}\right)^{-2 / y_{K}} Q_{l}(1.5)
$$

should be periodic in the variable $\ln \left(r-r_{0}\right)$ with period $y_{K} \ln 2$. In other words, $f_{1}\left(r-r_{0}\right)$ must be unchanged if $r-r_{0}$ is changed by a factor $2^{y} K$, in this case a factor of about 3. However there is no requirement that $f_{1}\left(r-r_{0}\right)$ be identically a constant. Nevertheless the numerical calculations showed that $f_{1}\left(r-r_{0}\right)$ is a constant independent of $r-r_{0}$, at least within the numerical errors of calculation (see Table III). The author has invented much

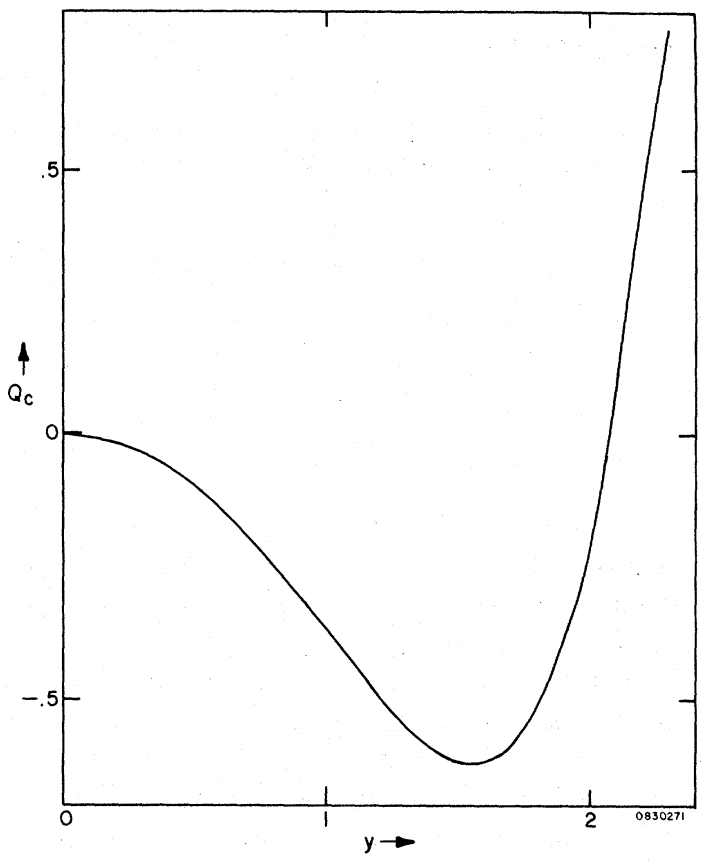

FIG. 4. The function $Q_{c}(y)$ vs $y$.

simpler recursion formulas which also have a fixed point and an unstable solution about the fixed point, for which an analogous function $f_{1}(\epsilon)$ can be defined, and found there also that $f_{1}(\epsilon)$ was strictly constant by numerical calculation, although the formal analysis requires only periodicity. The author has no explanation for this result.

\section{LOOSE ENDS}

This completes the detailed discussion of the recursion formulas. Many questions remain, but

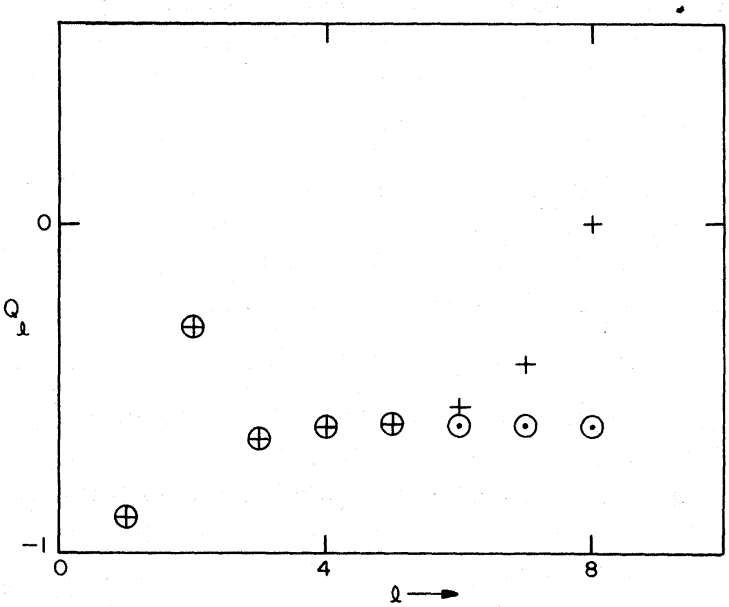

FIG. 5. Plot of $Q_{l}(1.5)$ vs $l$ : Crosses are for $r$ -1.66673 , circles for $r \simeq-1.66678$. In the latter case $Q_{l}(1.5)$ was $\simeq-0.62$ until $l=21$. 
TABLE III. Values of $A_{t}=2^{-l y_{K}}\left[Q_{t}(1.5)-Q_{c}(1.5)\right] /$ $\left(r-r_{c}\right)$ and $B_{l}=2^{-2 l}\left(r-r_{c}\right)^{-2 / y_{K}} Q_{l}(1.5)$ vs $l$, for $r-r_{c}$ $=0.00005$ and $r-r_{c}=0.000033$. The value chosen for $y_{K}$ is 1.65. One expects $A_{l}$ to be constant and independent of $r$ for intermediate values of $l$ while $B_{l}$ should be constant for large $l$.

\begin{tabular}{rrrrr}
\hline & \multicolumn{2}{c}{$r-r_{c}=5 \times 10^{-5}$} & \multicolumn{2}{c}{$r-r_{c}=3.3 \times 10^{-5}$} \\
\hline$l$ & $A_{l}$ & $B_{\imath}$ & $A_{l}$ & $B_{l}$ \\
3 & -18.3 & -1720 & -28.5 & -2855 \\
4 & -0.28 & -412 & -1.15 & -686 \\
5 & 1.14 & -100 & 1.00 & -168 \\
6 & 1.37 & -23 & 1.35 & -40 \\
7 & 1.41 & -4.3 & 1.40 & -8.3 \\
8 & 1.43 & 0.1 & 1.42 & -0.81 \\
9 & 1.49 & 0.98 & 1.46 & 0.82 \\
10 & 1.63 & 1.08 & 1.57 & 1.07 \\
11 & 1.89 & 1.05 & 1.78 & 1.06 \\
12 & 2.31 & 1.01 & 2.14 & 1.02 \\
13 & 2.90 & 1.00 & 2.66 & 1.00 \\
14 & 3.67 & 0.99 & 3.37 & 0.99 \\
15 & 4.68 & 0.98 & 4.28 & 0.98 \\
\hline \hline
\end{tabular}

hopefully the present paper is an adequate introduction to the phase-space cell method of analysis.

There are six loose ends to be mentioned. One is the problem of an external field. A term $h \sum_{\vec{n}} s_{\vec{n}}$ in the original interaction cannot be written in terms of the phase-space cell variables $s_{l \overrightarrow{\mathrm{m}}}$ because $\sum_{\text {in }} s_{\text {i }}$ corresponds in momentum space to $\sigma_{\mathbb{E}}$ with $\overrightarrow{\mathrm{k}}=0$. The point $\overrightarrow{\mathrm{k}}=0$ is excluded from all the momentum shells with finite $l$. So to accommodate this term one must redefine the momentum-space division to have only a finite number of shells, with the innermost shell replaced by a sphere. That is, the variable $l$ has a maximum $l_{\max }$ and the momentum-space cell for $l=l_{\max }$ is the sphere $|\overrightarrow{\mathrm{k}}|$ $\leq 2 \times 2^{l_{\max }}$. There are two ways to justify this redefinition. One way is to put the system in a box of volume $V$, in which case no momentum-space cell can have volume less than $V^{-1}$. Then $l_{\max }$ is (roughly)

$$
2^{d l_{\max }}=V .
$$

The other way to justify a finite $l_{\max }$ is if the system has a finite correlation length $\xi$; then one can choose $l_{\max }$ by

$$
2^{l_{\max }}>\xi
$$

This is possible because when $2^{t}>\xi$, the positionspace boxes associated with shell $l$ are larger than the correlation length and coupling between these boxes should be small. Hence there is no need to restrict the momentum $\overrightarrow{\mathrm{k}}$ to a shell instead of a sphere in order to reduce the coupling between these boxes. Since $\xi$ depends on $K$ and $h$, this means the allowable values of $l_{\text {max }}$ also depend on $K$ and $h$. This complicates the analysis which is why only the case $h=0$ was discussed in detail in this paper.

The second problem is the question whether $\eta$ will be different from zero in more accurate calculations. One correction that could change $\eta$ arises as follows. In the integral of $P[s(\overrightarrow{\mathrm{x}})]$ over the box $\overrightarrow{\mathrm{m}}$ one replaced $s^{\prime}(\overrightarrow{\mathrm{x}} / 2)$ by $s^{\prime}\left(\overrightarrow{\mathrm{m}} a_{0} / 2\right)$. A better calculation would replace $s^{\prime}(\overrightarrow{\mathrm{x}} / 2)$ by a Taylor's series about $\overrightarrow{\mathrm{m}} a_{0} / 2$ :

$s^{\prime}(\overrightarrow{\mathrm{x}} / 2)=s^{\prime}\left(\overrightarrow{\mathrm{m}} a_{0} / 2\right)+\frac{1}{2}\left(\overrightarrow{\mathrm{x}}-\dot{\mathrm{m}} a_{0}\right) \cdot \nabla s^{\prime}\left(\overrightarrow{\mathrm{m}} a_{0} / 2\right)+\cdots$.

If the terms involving $\nabla s^{\prime}\left(\overrightarrow{\mathrm{m}} a_{0} / 2\right)$, etc., are kept one has the possibility of generating extra terms proportional to $\left[\nabla s^{\prime}\left(\overrightarrow{\mathrm{m}} a_{0} / 2\right)\right]^{2}$ in calculating the integral over $s_{\overrightarrow{\mathrm{m}} 0}$. This would change the recursion formula for $K_{i+1}$ [Eq. (3.40)]; one can show that changes in this recursion formula lead ultimately to possible changes in $\eta$.

The third problem is the question of what happens in two dimensions. The author was unable to get sensible results from the recursion formula in two dimensions. The reason is that the recursion formula has a stabilizing feature in three dimensions and higher which is absent for two dimensions. The stabilizing feature is that in three dimensions and higher $2 \times 2^{-d / 2} z$ is less than $z$. As a result, $Q_{l+1}(z)$ for $z$ large is determined by the behavior of $Q_{l}(y)$ for $y<z$ [cf. Eqs. (3.41) and (3.43)]. Hence the asymptotic behavior of $Q_{0}(y)$ for large $y$ has little influence on the function's $Q_{l}(z)$ for large $l$ and fixed $z$. This is no longer true in two dimensions, and this makes the solutions of the recursion formulas be erratic in two dimensions. Hopefully this situation can be cured by taking into account terms which make $\eta$ nonzero; this could restore stability. The author has not checked this hypothesis in detail.

The fourth problem is why there is no discussion here of low temperatures $\left(T<T_{c}\right)$. There are two reasons for this. One is that for low temperatures the initial nearest-neighbor coupling constant $K$ is larger than the critical coupling; as a result it may no longer be legitimate to neglect the coupling between different position-space boxes in the phasespace cell analysis. Another reason is that, below the critical temperature, numerical calculations indicate that the functions $Q_{l}(y)$ become sharply peaked as $l$ increases. As a result the crude square-wave approximation to $\psi_{\overrightarrow{\mathrm{m}} l}(\overrightarrow{\mathrm{x}})$ may no longer be adequate; the approximation of replacing $s^{\prime}(\vec{x})$ by a constant may also be poor. (I thank G. Golner for discussion on this point.)

The fifth problem is whether the Widom-Kadanoff scaling laws are valid for the qualitative analysis of this paper. In I it was shown that these scaling laws could be derived from the renormalization group equations. However, in I it was assumed that the renormalization-group equations are exact 
even away from the critical point. The analysis of this paper is, so far, restricted to zero magnetic field above the critical temperature, and this is not sufficient for deriving the scaling laws. It may be that one can extend the analysis sufficiently to be able to establish the scaling laws, but this the author has not done.

Finally, there is the question why the momentum shells were chosen to include exactly a factor-of-2 range in momentum. Why not a factor 1.5 or 3 instead? The factor 2 represents a compromise between two conflicting desires. On the one hand one wishes to make the factor as small as possible to reduce the coupling between neighboring boxes in position space. If one keeps all momenta below the limit $2 \times 2^{-t}$, i. e., replaces the shell by a sphere, then the coupling is critical (so long as $\left.2^{i}<\xi\right)$. So it seemed that a minimum reduction would be to remove half the range $0 \leq k \leq 2 \times 2^{-l}$ giving the shell $2^{-l} \leq k \leq 2 \times 2^{-l}$; i. e., the factor 2 is an upper bound to what one can accept. On the other hand one wants the position-space boxes associated with shell $l+1$ to be as large as possible relative to the boxes for shell $l$ to justify neglecting the $\vec{x}$ dependence of $s^{\prime}(\vec{x} / 2)$ over the $l$ th box. Again a factor of 2 in size seemed the minimum acceptable, but this means the radius of the $(l+1)$ th. momentum shell has to be no more than half the radius of the $l$ th shell. So the factor of 2 is a lower bound. I cannot argue that 1.9 or 2.1 would be worse than 2 , but it is ridiculous to substitute 1.9 or 2.1 for 2 in an analysis as crude as this. The author has not tried varying this factor in the numerical calculations.

\section{CONCLUSIONS}

The phase-space cell analysis of this paper extends the Kadanoff block picture ${ }^{2}$ in several respects. First, it was possible to obtain explicit exponents from the phase-space cell analysis $(\nu=0.61, \gamma=1.22$, and $\eta=0$; with more effort one could derive the other exponents). These exponents are not exact, but their errors from the expected values for the Ising model are small. The smallness of the error may well be fortuitous, but even so there is much to be learned from the analysis of this paper. Secondly, one sees how one can motivate the existence of block Hamiltonians. In this paper the block Hamiltonian with block size $L$ arises as a result of integrating the components of the spin with momenta above $L^{-1}$. The interpretation of the resulting effective interaction as a block Hamiltonian is a qualitative interpretation. The block Hamiltonian is not truly a function of blocks of spins with sharp boundaries; instead, it is a function of an effective spin field $s_{L}(x)$ which has fluctuations only for distances of order $L$ or larger. Thirdly, the block Hamiltonians are not effective
Ising models with only two parameters $K_{L}$ and $h_{L}$; the effective interactions of this paper have the Landau-Ginsberg form and contain an unrestricted function $P_{L}(s)$ which is equivalent to an infinite number of parameters. Thus the Kadanoff picture has been generalized to allow for a continuous spin variable and for a more general form of interaction. Still, the basic qualitative features of the Kadanoff picture are maintained in this generalization. The recursion formula connecting $\mathcal{K}_{l}$ to $\mathcal{H}_{l+1}$ (which is equivalent to doubling the block size) is analytic, just as the Kadanoff picture predicts (see I). At the critical temperature the effective interaction approaches a fixed point of the recursion formula, just as was predicted from the Kadanoff picture (although this prediction was not guaranteed to work in the presence of irrelevant variables). The critical exponents are related to solutions of the linearized form of the recursion formula, just as was the case for the Kadanoff picture.

The importance of the phase-space cell analysis is that it provides an overview of the problem of how critical behavior arises from the partition function examined in this paper. By studying the phase-space cell analysis one gets many ideas on how one has to set up an approximate calculation of the partition function in order to get a reasonable approximation to its critical behavior. The model calculation of this paper is not, itself, a satisfactory approximation because one cannot trust the assumptions made in deriving the model. But maybe one can learn enough from this model so that one can set up sensible and reliable approximations that can be trusted.

What are some of the ideas that one learns from the phase-space cell model?

First, there is the idea of introducing the momentum shells and then separating the calculation of the partition function into integrations over the spins $\sigma_{\overrightarrow{\mathbf{k}}}$ for each momentum shell. The advantage of this is that the integration over a single momentum shell gave analytic results, so it is reasonable to introduce approximations in the calculation of these integrals. Along with the momentum-shell division, it is crucial that one makes the same approximations for every momentum shell, at least for momenta in the range $\xi^{-1} \ll k \ll 1$. That is, the recursion formula that one gets from integrating out a particular momentum shell $l$ should be independent of $l$. Otherwise it is hard to set up the formal theory of the fixed point and the relation of the critical exponents to departures from the fixed point. It was natural, in our analysis, to make the same approximations for all momentum shells. This was true because the degree of freedom $s_{\mathrm{mi}}$ were defined so that there were separate degrees of freedom for each momentum shell $l$, and because the degrees of freedom for different shells 
were related by a scale transformation. Thus once the recursion formulas were derived for one momentum shell, only a rescaling was needed to get the recursion formulas for all momentum shells.

It is evident from the phase-space cell analysis that one must approximate reasonably well all the momentum-shell integrations with $\xi^{-1}<k<1$. Any serious error for any momentum in this range will disturb the process by which the interaction $\mathfrak{H}_{l}$ [i.e., $Q_{l}(z)$ ] first approaches the critical interaction and then (for large enough $l$ ) goes away from the critical interaction as one integrates down to $k \sim \xi^{-1}$. In a renormalization-group approach one treats all momentum shells uniformly and so one's approximations are uniformly good (or bad) for all shells. If one does not separate the degrees of freedom for different momentum shells, it is quite likely that one's approximations will favor some momentum shells over others, resulting in a poor description of critical behavior.

A second idea in the phase-space cell analysis is that of reducing the interactions between degrees of freedom to a manageable level by appropriately defining the degrees of freedom. What is a manageable level? In the model of this paper the couplings between degrees of freedom have been simplified so that when one integrates over a particular variable $s_{\overrightarrow{\mathrm{m}} l}$, only one parameter $z$ need be specified [as in the integral for $I_{l}(z)$, Eq. (3.41)]. This is clearly a manageable level. In the original form of the partition function [Eq. (1.2)], if one tries to integrate successively all the spins $s_{\vec{n}}$, one will find that after the first few integrations each remaining $s_{\text {if }}$ is coupled to many other spins. It is then hopeless to do any further integration because each integral will involve a large number of other spins, and the integral over any given spin must be computed as a function of all the other spins involved. The way the simplification was achieved was to reduce the coupling between adjacent position-space cells at the price of introducing separate momentum shells and thereby having couplings between these shells. The momentum-space shell couplings (after some simplification) turned out to be manageable. For a more precise calculation the couplings between position-space cells would have to be considered but hopefully these would be taken into account in a perturbation expansion. In any case one has to arrange the calculation of the partition function so that any given integral involves only 1 or 2 other variables; an integral involving 5 or 10 or more other variables is unmanageable. The hardest challenge in developing a quantitative formulation of the renormalization group is to meet this requirement. A renormalization-group framework helps one to meet this requirement in that if one can design the calculation for one momentum shell to meet the requirement, then the calculation for all other momentum shells will also meet the requirement.

The phase-space cell analysis provides an explicit answer to the question raised at the beginning of I. Namely, given that the partition function is simply a set of integrations over an analytic function of $K$ and $h$, how can the partition function (or, to be precise, the free energy) be singular in these variables? One finds in the phase-space cell analysis that any particular integration produces analytic results; that is, the function $Q_{l+1}(y)$ appearing in the interaction $\mathfrak{F}_{l+1}$ depends analytically on any parameters appearing in $Q_{l}(y)$. The nonanalyticity in the partition function arises because there are an infinite number of momentum shells to be integrated over so one has to iterate the recursion formula an infinite number of times to calculate the partition function. The recursion formula is analogous to a time translation over a unit time interval for a classical particle in a well-behaved potential. The position of the particle after one unit of time is an analytic function of its initial position; but if one has to repeat the time translation an infinite number of times, the analyticity can be lost. This was explained in detail in I.

To conclude this paper the term "renormalization group" will be explained. It is not necessary to understand the meaning of this term: It may be regarded as simply a code name for the transformations discussed in these papers. But one can see that this term does make sense. When one replaces the spin variable $s_{l}(\overrightarrow{\mathrm{x}})$ by the scaled variable $s_{c l}(\overrightarrow{\mathrm{x}})$ one renormalizes the spin by the factor $2^{-l d / 2} \alpha_{0} \cdots \alpha_{l-1}$ [see Eq. (3.50)]. This is where renormalization is involved in a literal sense. Figuratively, field theorists use the term renormalization to mean also the changes that take place in an interaction when one changes a cutoff; analogous to this are the changes in the effective interaction $\mathfrak{H}_{l}$ which result from changing the block size (i. e., changing $l$ ). The group is the group of transformations generated by iterating the recursion formulas. These formulas can be thought of as defining a transformation $T$ on a function space of functions of one variable $Q(y)$. Iterating the recursion formulas defines the transformations $T^{2}, T^{3}$, etc. If the renormalization group is defined in terms of an infinitesimal transformation, as in $I$, it is easy enough to define the inverse transformation $T^{-1}$ (corresponding to decreasing the block size by a factor 2). In this paper it is not clear that an inverse transformation exists. It does not matter since we had no need to use the inverse transformation.

\section{ACKNOWLEDGMENTS}

I wish to thank Professor M. Fisher, Professor B. Widom, Professor L. Kadanoff, Professor D. 
Jasnow, and Professor M. Wortis for many invaluable conversations. I am grateful to Dr. P. Hohenberg, Professor P. Martin, and others at the Midwinter Conference on Solid State Physics at Irvine (1970) for discussion. I thank Dr. A.
Suri for discussions. I thank Professor J. Wilkins and Dr. K. Murata for explaining the Kondo problem to me, and Professor E. B. Wilson, Jr. and Dr. K. Piech for discussions of the problems of atoms and molecules.
* Supported in part by the National Science Foundation. ${ }^{1} \mathrm{~K}$. Wilson, preceding paper, Phys. Rev. B $\underline{4}, 3174$ (1971), referred to as I hereafter.

${ }^{2}$ L. P. Kadanoff, Physics 2 , 263 (1966); L. P. Kadanoff et al., Rev. Mod. Phys. 39, 395 (1967).

${ }^{3}$ For a review of critical phenomena in the Ising model and other theories, see M. E. Fisher, Rept. Progr. Phys. 30, 731 (1967). See also L. P. Kadanoff et al. (Ref. 2).

${ }^{4}$ The precise definition of $K_{L}$ and $h_{L}$ is given in I.

${ }^{5}$ V. L. Ginsberg and L. D. Landau, Zh. Eksperim. i Teor. Fiz. 20, 1064 (1950). See, also J. Schrieffer Superconductivity (Benjamin, New York, 1964), p. 19.

6"Irrelevant variables" were defined in I.

${ }^{7}$ The critical exponents $\gamma, \nu$, and $\eta$ are as defined in Ref. 3; definitions are also given later in this paper. The other critical exponents will not be computed here, nor will a full derivation of the scaling laws be obtained. This is because the detailed analysis reported in this paper is limited to the case of no external magnetic field and temperatures above the critical temperature. For a discussion of this limitation see Sec. V.

${ }^{8}$ K. Wilson, Phys. Rev. 140, B445 (1965); see also Phys. Rev. D 2 , 1439 (1970).

${ }^{9} \mathrm{~K}$. Wilson, Cornell report No.CLNS-142 (unpublished); this reference summarizes some of the ideas which will be discussed in detail in the present paper. The Feynman path integral discussed in this reference is identical to the partition function discussed here: The functional integral over $A[\phi]$ defined in Eq. (9) of this reference (with a cutoff field $\phi$ ) is mathematically identical to the functional integral over $e^{x c}$ using the Landau-Ginsberg form $(1,1)$ for $\mathcal{F}_{L}$. The same identity between critical phenomena and field theory appears in A. A. Migdal, Zh. Ek- sperim. i Teor. Fiz. 59, 1015 (1970) [Sov. Phys. JETP $32,552(1971)$ ], and references cited therein; see also A. M. Polyakov Zh. Eksperim. i Teor. Fiz. 59, 542 (1970) [Sov. Phys. JETP, 32, 296 (1971)].

${ }^{10}$ T. H. Berlin and M. Kac, Phys. Rev. 86, 821 (1952). The spherical model is also discussed in this reference. The spherical model is distinct from the model of Eq. (1.2) because the spherical model imposes a restriction on the quantity $\sum_{\vec{n}} s_{\mathbb{n}}^{2}$; there is no such restriction in Eq. (1.2). The extension of the spherical model to include a quartic term has been discussed by J. Langer, Phys. Rev. 137, A 1531 (1965).

${ }^{11}$ While the magnitude $\left|\phi_{\vec{m}}(\overrightarrow{\mathrm{k}})\right|$ can be roughly approximated by a constant, $\phi_{\overrightarrow{\mathrm{m}}}(\overrightarrow{\mathrm{k}})$ itself cannot. The reason is that if the cell $\overrightarrow{\mathrm{m}}$ is far from the origin in position space then $\phi_{\overrightarrow{\mathrm{m}}}(\overrightarrow{\mathrm{k}})$ will have a phase which changes rapidly with $\overrightarrow{\mathrm{k}}$.

${ }^{12}$ The variable $s_{\overrightarrow{\mathrm{m}} l}$ is defined without a prime for legibility reasons.

${ }^{13}$ See Sec. V for a brief discussion on nonzero $h$.

${ }^{14}$ See Ref. 3 for a definition of the correlation function.

${ }^{15}$ See L. Landau and E. M. Lifschitz, Quantum $\mathrm{Me}$ -

chanics (Pergamon, London, 1958), pp. 164 and 185.

${ }^{16}$ To be precise one must substitute $Q_{l+1}$ for $Q_{l}$ in Eq. (3.41) for this calculation.

${ }^{17}$ The quantity $R_{l}\left(2 \times 2^{-d / 2}(z-y)\right)$ can occur also.

${ }^{18}$ See Ref. 3.

${ }^{19}$ See Sec. V.

${ }^{20}$ This is true in the absence of a magnetic field; the author has not worked through the problem with a magnetic field present.

${ }^{21}$ See, e.g., F. Hildebrand, Introduction to Numerical

Analysis (McGraw-Hill, New York, 1956), Chap. 8.

${ }^{22}$ See Ref. 3. 